\title{
TUTORIAL
}

\section{Validation of nonlinear gyrokinetic transport models using turbulence measurements}

\author{
A. E. White $\dagger$ \\ Massachusetts Institute of Technology, Cambridge, MA 02139, USA
}

(Received 2 April 2018; revised 14 November 2018; accepted 16 November 2018)

This tutorial covers validation of gyrokinetic turbulent-transport models via comparison of measured turbulence with realistic simulations of fusion plasmas. It presents a brief history of validation of gyrokinetic simulations, the principal challenges encountered, a limited survey of common turbulence diagnostics used on tokamaks and stellarators, an overview of the fundamentals of synthetic diagnostic models and a discussion of frontiers in turbulent-transport model validation.

Key words: fusion plasma, plasma diagnostics, plasma simulation

\section{Introduction}

Comparing gyrokinetic simulation results to experimental results via a rigorous methodology (Terry et al. 2008; Greenwald 2010) forms the area known as gyrokinetic validation within the fusion energy research field. The drift-wave turbulence observed in the core of fusion plasmas is well described by the nonlinear gyrokinetic equations coupled to Maxwell's equations (Brizard \& Hahm 2007; Krommes 2010); nonlinear gyrokinetic theory is encapsulated in numerical gyrokinetic codes (Garbet 2010). The physics of drift-wave turbulence (Horton 1999; Weiland 1999) in tokamak and stellarator plasmas has been extensively developed for many years. This effort is motivated by the desire to more accurately describe the higher than observed levels of cross-field heat and particle transport that occur in fusion plasmas. In the core region of fusion plasmas, $0.0<r / a<0.9$, where $r$ is the minor radius and $a$ is the plasma radius, the drift-wave instabilities and associated low frequency turbulence (with $\omega \ll \Omega_{\text {ci }}$ ), have small amplitudes with respect to the background densities and temperatures (e.g. $\tilde{n} / n \sim 1 \%$ ), and correlation lengths in the range of $\sim 0.1-10 \rho_{\mathrm{s}}$. Here $\Omega_{\mathrm{ci}}$ is the ion cyclotron frequency and $\rho_{\mathrm{s}}$ is the ion sound gyroradius. Drift-wave instabilities are in general electromagnetic and occur at multiple scales, spanning a range of normalized wavenumbers $0<k_{\theta} \rho_{\mathrm{s}}<60$, where $k_{\theta}$ is the poloidal wavenumber of the fluctuations. The drift-wave turbulence described by nonlinear gyrokinetic theory leads to particle fluxes $\Gamma_{\mathrm{j}}=\left\langle\tilde{n}_{\mathrm{j}} \tilde{v} E_{\mathrm{r}}\right\rangle+n_{\mathrm{j}}\left\langle\tilde{v}_{\mathrm{j} \|} \tilde{B}_{\mathrm{r}}\right\rangle / B$ and energy fluxes $Q_{\mathrm{j}}=3 / 2\left\langle\tilde{p}_{\mathrm{j}} \tilde{v} E_{\mathrm{r}}\right\rangle+\left\langle\tilde{q}_{\mathrm{j} \|} \tilde{B}_{\mathrm{r}}\right\rangle / B+5 / 2 p_{\mathrm{j}}\left\langle\tilde{v}_{\mathrm{j} \|} \tilde{B}_{\mathrm{r}}\right\rangle / B$, where subscript $\mathrm{j}$ denotes the species (electrons or ions). The turbulent fluxes depend on the amplitude of fluctuations of

$\dagger$ Email address for correspondence: whitea@mit.edu 
the density $\tilde{n}$, pressure $\tilde{p}$, the parallel heat flux $\tilde{q}_{\|}$, parallel velocities $\tilde{v}_{\|}$, electric field $\tilde{E}_{\mathrm{r}}$ and magnetic field $\tilde{B}_{\mathrm{r}}$. The fluxes also depend on the mean of the relative phases between the different fluctuations. The brackets denote ensemble averages of fluctuating quantities (Conway 2008).

The complex interplay among electromagnetic, multi-scale drift-wave turbulence and the background equilibrium flows $(E \times B$ shear) and self-generated flows (zonal flows and geodesic acoustic modes) in fusion plasmas forms a 'standard' turbulent-transport paradigm. This standard paradigm of turbulent-transport is part of the physics basis for ITER (Doyle 2007). Over the last two decades, advances in numerical techniques have allowed for the development of high-fidelity gyrokinetic turbulence simulation codes (such as GENE (Jenko et al. 2000; Görler et al. 2011) and GYRO (Candy $\&$ Waltz 2003a) and many others) that are appropriate for direct and quantitative comparison with experiment. Gyrokinetic validation is used to test and extend the standard turbulent-transport paradigm as it is implemented in such codes in order to develop a deeper understanding of plasma turbulence and to advance predictive capabilities for fusion energy development.

General validation approaches in fusion energy research has been described in review articles (Terry et al. 2008; Greenwald 2010). Validation of gyrokinetic codes specifically using validation metrics has also been reviewed in depth (Holland 2016). This tutorial is intended to provide readers with a working knowledge of experimental steps necessary to compare nonlinear gyrokinetic codes with measurements of turbulence in tokamaks and stellarators. This tutorial provides descriptions of the experimental data used as input to gyrokinetic codes, as well discussion of the different turbulence parameters that can be measured in the core of fusion plasmas. The philosophy and workflow for comparing these measurements to nonlinear gyrokinetic simulation results is described.

This tutorial will focus primarily on using gradient-driven, local $\partial f$ simulations for comparisons with measured turbulence. Local, gradient-driven $\partial f$ gyrokinetic simulations have been used most extensively to date for comparisons with turbulence measurements. Published methodologies and best practices for gyrokinetic validation have discussed benefits of local, gradient-driven simulations for comparisons with experiment (Holland 2016).

In the $\partial f$ formulation, a separation between turbulent scales and equilibrium profile scales is assumed, which is found to be a good assumption in the core plasma of medium-scale fusion devices (Barnes et al. 2010; Holland et al. 2011; Jenko et al. 2013), when there is a sufficiently small ratio of ion Larmor radius to plasma minor radius, or $\rho^{*}$. More details of the assumptions used in the $\partial f$ formulation used in gyrokinetic codes is described in Garbet (2010), and that article also provides a clear discussion of the effects of geometry, and benefits of both local models and global models. Because $\partial f$ codes exploit the large scale separation between the spatiotemporal scales of the turbulence and the background pressure profiles, they can only model the effect the pressure profiles have on the turbulence, they do not model how the turbulence affects the evolution of the background profiles. In contrast, in a full- $f$ global gyrokinetic code, the equilibrium and fluctuations are solved for self-consistently, assuming no scale separation (Garbet 2010). It is important to note that some $\partial f$ codes, like GYRO and GENE, can be run both locally and globally (Holland et al. 2011; Jenko et al. 2013). Performing a 'global run' with the $\partial f$ codes typically refers to how the boundary conditions are treated in the simulation domain and whether the background profiles are allowed to vary spatially in the domain. This would be in contrast to a fully global treatment, where the background profiles 
evolved in time. Good agreement is found between the local and global simulations for both GENE and GYRO (Holland et al. 2011; Jenko et al. 2013), when $\rho^{*}$ is sufficiently small in the core region of medium-size to large-size fusion plasmas.

This tutorial is limited in that it does not attempt to review the entirety of physics results obtained from gyrokinetic validation studies in the literature. This tutorial is intended to be code and model agnostic; however, as mentioned earlier, the majority of practical examples are drawn from experience with gradient-driven simulations, rather than flux-driven simulations (Garbet 2010); though the latter are discussed. In addition, the details of numerical methods used in gyrokinetic codes, such as how collisions, $E \times B$ shear or boundary conditions are implemented, are outside the scope of this paper. These details are of course highly relevant for both model validation and verification. Keep in mind that a necessary condition for validation is to use a verified code (Greenwald 2010); and validation and verification are not the same. For gyrokinetic transport models, verification is performed mostly via code-to-code comparisons, or benchmarking exercises (Bravenec et al. 2013; Merlo et al. 2018; Mikkelsen et al. 2018) and is not covered in this tutorial. On the experimental side, a much broader review of data analysis and data validation in fusion plasmas is presented by Arshad et al. (2008) and a review of turbulence measurements and data analysis techniques is found in Tynan, Fujisawa \& McKee (2009).

This paper is organized as follows: $\S 2$ presents a brief history of gyrokinetic transport model validation and best practices, focusing on the use of fluctuation measurements. This section discusses challenges particular to validation of gyrokinetic transport models, and provides a practical workflow-level view of validation from an experimental perspective. Section 3 describes the analysis steps required for preparing experimental data for comparisons with gyrokinetic codes. Section 4 provides a survey of several common turbulence diagnostics that have been used in gyrokinetic validation studies over the past decade, describing their fundamental operational principles and basic data analysis techniques. Section 5 presents an overview of synthetic diagnostics, which are needed to quantitatively compare turbulence measurements to gyrokinetic simulations. Section 6 presents several open questions in validation and examples of work at the frontiers of the field, with $\$ 7$ concluding with a discussion and outlook for the field.

\section{Gyrokinetic transport model validation and best practices}

\subsection{Brief history of gyrokinetic model validation}

There have been many milestones in gyrokinetic transport model validation in the last two decades. Here, only a few are highlighted as they pertain to defining the challenges and actionable aspects of validation using turbulence measurements. One important result is from Lin et al. (1998), which demonstrated that the interaction between zonal flows (Diamond 2005) and turbulence is a nonlinear saturation mechanism that has important implications for reducing turbulent amplitudes and associated heat flux. A second milestone of interest was that of Candy \& Waltz $(2003 b)$, which demonstrated that nonlinear gyrokinetic codes could match both the experimental ion and electron transport levels within error bars, when sheared equilibrium $E \times B$ shear rotation (Burrell 1998) effects were included in the model. Around the same time, radial correlation lengths obtained using reflectometer measurements were first compared with gyrokinetic simulations (Rhodes et al. 2002). The results showed that self-generated zonal flows were required to reduce the simulated correlation length of the turbulence, bringing it into agreement with the 
experimental values. Another paper that same year described comparisons between gyrokinetic simulations and experimental ion and electron heat fluxes, and also turbulent fluctuation levels measured with a beam-emission spectroscopy (BES) diagnostic (Ross \& Dorland 2002). The results showed that including $E \times B$ shear was important to reduce the heat flux and turbulent levels nearer the experiment, but it was not possible to match the experimental values within error bars.

The specifics of the agreement and disagreement in these 'first validation papers' (Rhodes et al. 2002; Ross \& Dorland 2002) are not what make these papers of interest in this tutorial. Instead, it is because the work includes many of the modern 'best practices' for validation, later described in review papers (Terry et al. 2008; Greenwald 2010; Holland 2016). Key concepts and best practices in validation include qualification of the domain of model applicability and limitations, identifying uncertainties in the model and the experiment, recognizing known deficiencies or excluded physics in the model, sensitivity analysis and use of the primacy hierarchy and validation metrics (Terry et al. 2008; Holland 2016). The papers (Rhodes et al. 2002; Ross \& Dorland 2002), were among the first to systematically scan code inputs within experimental error bars to test model sensitivity. These papers also used many validation observables in a primacy hierarchy (though did not call it by this name) to compare not just to heat fluxes inferred from power balance, but also measured turbulence, to the simulations. These papers also featured the description and use of multiple transport models. Within a gyrokinetic code, the user can turn on and off different physics effects within the same code. This allows the validation concepts of qualification and deficiency of models to be addressed. It is more appropriate to discuss validation of transport models (which can be run using the same code or different codes), rather than validation of a transport code.

For validation of the gyrokinetic turbulent-transport model, numerical codes must be used, which adds to the complexity of making these comparisons. Figure 1 shows an abstract diagram of the validation process, which is iterative, and ideally combines experimental and simulation planning in parallel. Perhaps the most important guiding principle is that the goal of a successful validation study should not be to achieve agreement between experiment and simulation. The purpose of a successful validation study is to understand the reasons for agreement or disagreement between the experiment and the model. Robust agreements and disagreements that can be understood in terms of physics in the model or measurement limitations etc., will lead to progress in development of new models and experiments, and ultimately, to deeper understanding of turbulent transport in fusion plasmas. Serendipitous agreements and accidental disagreements do not aid scientific progress. Reporting robust disagreement is just as important as reporting robust agreement. While not an exhaustive list, the following selected papers from the last decade contain several good examples of the 'best practices' in validation put to use for comparisons between simulation and experimental ion and electron heat transport, impurity transport and a variety of turbulence measurements in L-mode, I-mode (Whyte et al. 2010) and H-mode plasmas in tokamaks: White et al. 2008; Casati et al. 2009; Holland et al. 2009; White et al. 2010; Rhodes et al. 2011; Holland et al. 2012; Howard et al. 2012; Told et al. 2013; Citrin et al. 2014; Field et al. 2014; Görler et al. 2014; Sung et al. 2016; Creely et al. 2017; van Wyk et al. 2017; Freethy et al. 2018; Howard et al. 2018.

\subsection{Designing gyrokinetic model validation experiments}

Dedicated validation experiments provide the best opportunities for rigorous comparison between experiment and theory, as encapsulated in a model. Carrying out 


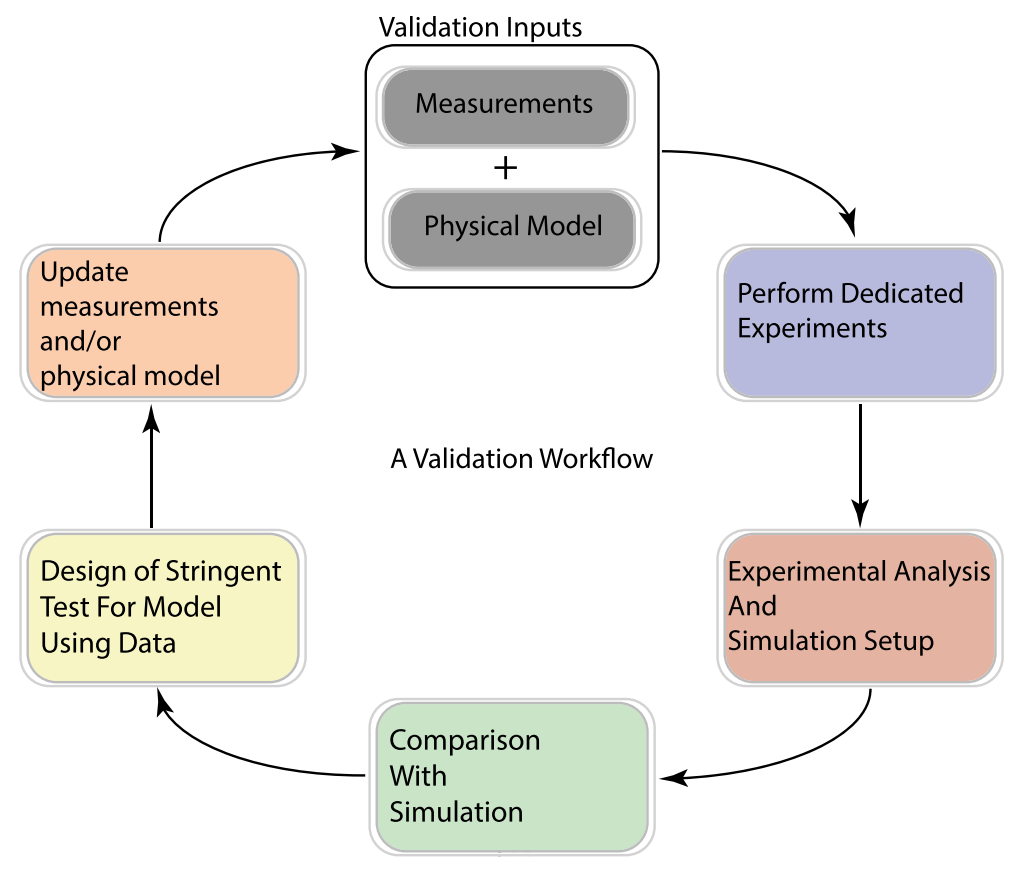

FIgURE 1. Validation of a model is a process. After a dedicated experiment is run, the experimental measurements of turbulence and transport (validation observables) are compared with outputs from the codes. Points of agreement and disagreement are assessed (ideally, quantified using validation metrics), and new models or and/or measurements are developed, and the cycle, or parts of it, is repeated.

a carefully controlled validation experiment in fusion plasmas and subsequent comparisons with gyrokinetic codes presents several challenges, a few of which are described below.

First, when planning an experiment to study drift-wave turbulence and transport in fusion plasmas, one will seek to isolate a particular physical effect in the model. For example, the drift-wave turbulence is highly sensitive to the primary driving gradient(s). Both the linear instabilities, or modes, and the turbulence associated with the nonlinearly saturated state, can be classified according to the dominant driving mechanism. Three primary instabilities of interest in the core plasma are the ion temperature gradient (ITG) mode, the electron temperature gradient (ETG) and the trapped electron mode (TEM). ITG modes are long-wavelength ion-scale $\left(k_{\theta} \rho_{\mathrm{s}}<1\right)$ instabilities that are driven by the ion temperature gradient. ETG modes are short-wavelength electron-scale $\left(k_{\theta} \rho_{\mathrm{s}}>1\right)$ instabilities that are driven by the electron temperature gradient. TEMs can span both long and short wavelengths, and are driven by electron temperature and density gradients. ITG, TEM and ETG modes can exist electrostatically, and persist when electromagnetic effects are included. A fourth type, the microtearing modes (MTMs), are long-wavelength ion-scale electromagnetic instabilities that are excited at high plasma beta and are driven by electron temperature gradients. The kinetic ballooning mode (KBM) can also play a role at high beta (Ishizawa et al. 2013) and the resistive ballooning mode (RBM) may become relevant in the edge region, especially in L-mode plasmas (Rogers, Drake \& Zeiler 1998). It is always good practice to perform a linear stability analysis survey in the plasmas 

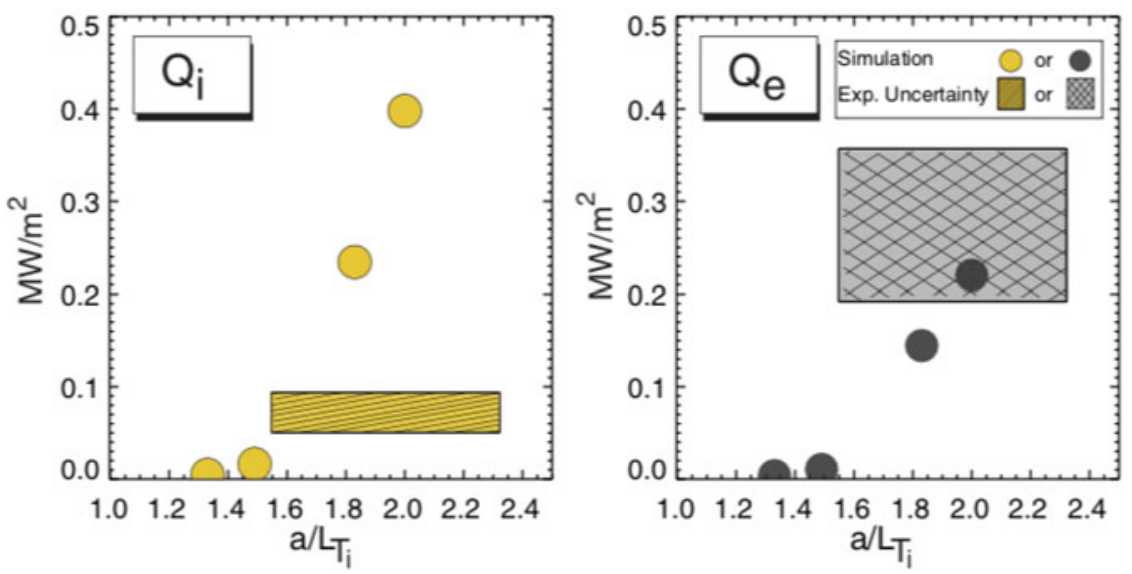

FIgURE 2. Nonlinear GYRO calculated output of ion heat flux, $Q_{\mathrm{i}}$, and electron heat flux, $Q_{\mathrm{e}}$, against the input values of $a / L_{\mathrm{Ti}}$, showing critical gradient behaviour and 'stiff' response in the flux-gradient curve, taken from figure 7 Howard et al. (2013).

of interest prior to nonlinear simulation, to help guide the set-up and execution of the nonlinear runs. In addition to the primary driving gradients, drift-wave turbulence is also very sensitive to the shape of the plasma, the magnetic shear, the $E \times B$ shear, etc. These effects have been explored using many simulations run over very large input parameter ranges, with examples shown in Kinsey, Waltz \& Candy (2006, 2007). These parameters can be controlled in tokamak and stellarator plasmas to some extent, but typically only over a limited range compared to simulation. And unlike in simulation, isolating the effect of one parameter is difficult (if not impossible) in the experiment.

Second, it is of interest to design dedicated validation experiments that minimize the uncertainties and errors on the measurements that the codes are using as input. Minimizing the errors is essential because it is well known that the nonlinear gyrokinetic model can be hypersensitive to input parameters (Holland 2016). For example, the error bar on an input parameter in a gradient-driven simulation, like the normalized logarithmic gradient of ion temperature, $a / L_{\mathrm{Ti}}$, may be $20 \%$. Within this range, the models can exhibit extreme sensitivity. This is because many of the modes exhibit a threshold in the gradient driving the instability: the mode is linearly stable below the threshold, or 'critical gradient', and unstable above. Nonlinearly, the saturated turbulence also exhibits a critical gradient, different from the linear value (Garbet 2010). Stiffness is defined in this paper the incremental change in flux for an incremental change in gradient above the critical gradient, which is a typical definition employed in gyrokinetic simulation studies (Citrin et al. 2014). Other definitions of stiffness and experimental investigations are described in the following references (Garbet et al. 2004; Ryter et al. 2006; Mantica et al. 2009; DeBoo et al. 2012).

In figure 2 nonlinear ion-scale gyrokinetic simulation output results at $r / a=0.5$ for two L-mode plasmas in C-Mod are shown. Details of the simulations can be found in Howard et al. (2013). The calculated output ion heat flux is plotted versus code input $a / L_{\mathrm{Ti}}$. Below the critical gradient, there is no heat flux driven, and above the critical gradient, the heat flux can increase dramatically, a factor of 10 increase in the output heat flux for a $10 \%$ change in the ion temperature gradient. These plasmas, like most experimental plasmas, are classified as being very 'stiff'. Sensitivity of the 
model outputs to the input driving gradient magnifies any experimental uncertainty. In figure 2 , the experimental error bars $( \pm 1$ sigma on the experimental ion heat flux, $Q_{\mathrm{i}}$ ) are marked by the shaded box. The error on the experimental value $a / L_{\mathrm{Ti}}$ is $1 \sigma= \pm 20 \%$. In this case the experimental gradients, within the measured error bars, bracket a range above and below the calculated nonlinear critical gradient. This makes simulation of experimental conditions quite challenging, and computationally much more expensive than simulations run with the strongly driven ITG dominant cyclone base case (Dimits et al. 2000), a common benchmarking case used to compare across codes. To address this hypersensitivity and the resulting uncertainties, developing rigorous validation metrics is essential (Holland 2016).

Some practical things to keep in mind with normalized parameters like the normalized logarithmic gradient of ion temperature, $a / L_{\mathrm{Ti}}$. First, gyrokinetic equations generally use the assumption that the gyroradius, $\rho$, is much smaller than the equilibrium scale length, $L$, that is, $\rho / L \ll 1$ (Weiland 1999). The inverse scale length, $1 / L$, is the logarithmic gradient of the temperature (or density, etc.), and for a Maxwellian distribution, appears in the diamagnetic drift frequency when one derives the linear gyrokinetic equation (Antonsen \& Lane 1980; Weiland 1999). In the literature, one will find that inverse scale length, $1 / L$, is sometimes normalized by the plasma minor radius, $a$, or by the machine major radius, $R_{0}$, or by the local value of the major radius, $R$; take care to identify which normalization the author is using.

Third, it is of interest to consider extreme cases motivated by theory, even though they cannot be tested directly. For example, in the cyclone base case (Dimits et al. 2000), the plasma is dominated by strongly driven ITG turbulence. Plasmas with only ITG-type turbulence can be studied numerically by setting $a / L_{\mathrm{Te}}=0$, and similarly only TEM-type turbulence can be studied by setting $a / L_{\mathrm{Ti}}=0$ in an ion-scale gyrokinetic simulation (Dannert \& Jenko 2005). It is not possible to create such a 'pure mode' state in experiments. Many experimental fusion plasmas will be 'mixed mode' over most of the core plasma, and when simulated, these plasmas are found to contain features of both types of turbulence (linear and nonlinear) within the range of experimental error bars considered for the gradient scale lengths, with one example being Sung et al. (2016). This can make it difficult to isolate physical effects that lead to agreement or disagreement with experiment in the validation study. Therefore, it is of interest to try to design a plasma that features dominant TEM turbulence (or ITG turbulence) (White et al. 2010; White et al. 2015; Ernst et al. 2016). Both linear and nonlinear gyrokinetic simulations can be used for predictive experimental design in such cases. The use of validation metrics can help discriminate between models to ultimately isolate physical effects (Holland 2016).

Fourth, it is important to measure as many different turbulence and transport parameters as possible to better assess and ideally quantify the agreement between the model and experiment. This motivates the use of turbulence measurements $(\S 4)$ and synthetic diagnostics $(\$ 5)$ for validation of nonlinear gyrokinetic codes. All the relevant physical quantities that can be measured or inferred (fluctuation levels, crossphase angles, correlation lengths, heat fluxes, etc.) become the validation observables, and are organized into a 'primacy hierarchy' (Terry et al. 2008; Greenwald 2010). The primacy hierarchy addresses several challenges, and in particular, helps to avoid fortuitous agreement (or disagreement) and helps to discriminate between models, especially in the case when the models are hypersensitive to input parameters. For gyrokinetic transport model validation, fluctuation levels of electron density and temperature, and their respective power spectra, tend to be measured most directly 
in experiments, and so are at the highest level in the primacy hierarchy. Correlation lengths and cross-phase angles are at the second level, because they require processing of two turbulence measurements. If turbulent particle, $\Gamma_{\mathrm{j}}$, or heat flux, $Q_{\mathrm{j}}$, of species $\mathrm{j}$ were to be measured in the experiment using combinations of fluctuation data, then these would be at the third level. These measurements are extremely challenging to make in the core plasma $(r / a<0.9)$ where the gyrokinetic model is most applicable. The inferred heat fluxes from power balance calculations would be classified as belonging at some very low level, because they are not measured directly. Defining a validation metric and applying it to the observables from experiments can be done in a variety of ways. One example from the TORPEX basic plasma experiments has been used to successfully discriminate between turbulence models (Ricci et al. 2011). The same metric has since been applied at ASDEX Upgrade to identify a nonlinear gyrokinetic simulation with the 'best match' to experiment, using inferred heat fluxes, electron temperature fluctuation levels, radial correlation lengths and the cross-phase angle between density and temperature fluctuations as the validation observables (Freethy et al. 2018). It is important to note that the strength of the primacy hierarchy and validation metric in these examples is discriminating between models, rather than assessing a single model in isolation; hence, from a practical perspective, planning for a validation experiment in a fusion device also means planning for computing resources needed to perform runs of different models with a gyrokinetic code.

From the perspective of an experimental approach, or workflow, for validation of gyrokinetic transport models, several general recommendations can be made: run experiments to obtain the highest quality of data used as input to verified and well-documented power balance codes and gyrokinetic codes, measure at many levels of the primacy hierarchy, measure turbulence at multiple radial locations, make multi-field fluctuation measurements (e.g. both $\delta T_{\mathrm{e}}$ and $\delta n_{\mathrm{e}}$ ) when possible, take care with quantification of uncertainties on experimental parameters, apply synthetic diagnostics, plan to use computing resources for extensive sensitivity scans with the codes both before and after an experiment, and use validation metrics.

\subsection{Example validation workflow}

Let us say that we wish to design an experiment to study how the turbulence and transport changes as the electron temperature gradients are varied. The profile changes will be actuated with electron cyclotron resonance heating (ECRH) power in an H-mode plasma. We want to use Doppler reflectometry (see §4) to measure the turbulence at several radial locations at each power level in the ECRH scan. Depending on the details of the Doppler reflectometer system, it may take one discharge at each power level to obtain the desired data set, or multiple discharges. It may be possible to change the power level during a long discharge and complete the desired Doppler reflectometer data set in a single shot. But keep in mind that for many fluctuation diagnostics, long, steady phases of the discharge will yield the best turbulence data. Prior to the experiment, development of a reliable synthetic diagnostic for the turbulence measurements may require a separate, possibly extensive, modelling project.

In addition to gathering the turbulence data, it is essential to also gather all the profile data required for comparisons with simulation; and long steady periods in the discharge will help reduce uncertainties on the measured background profiles. Of course, this type of planning is done for any experiment, and is not unique to 
a dedicated validation experiment. What is unique to a validation experiment is the interweaving of simulation planning and experiment planning from the beginning. This helps to guarantee that the comparisons with gyrokinetic codes will lend themselves to application of a rigorous validation methodology.

Once the experiment is designed, plans can be made for the steps required in the workflow, which will require both experimental data analysis and preparatory scoping scans with the codes. An example workflow based on using a gradient-driven gyrokinetic code to compare with experimental fluctuation measurements is presented in this section. Use of different codes (like a flux-driven code) will require a different workflow, in which case certain steps may be omitted and new steps may be added, but the spirit of the process remains the same.

Step 1: Identify a target discharge where high quality Doppler reflectometry (DR) was obtained previously. Check that the plasma parameters are within the domain of the model applicability for the model to be tested.

Step 2: Check that the target discharge also allows for high quality equilibrium reconstruction, ion and electron profile measurements, density profile measurements, rotation measurements, current profile measurements, etc. If additional turbulence measurements can be obtained in the same discharge, include them in planning as well. Obtaining as many measurements as possible, the validation observables, is important for later application of a validation metric.

Step 3: Check that the target discharge will stay in H-mode during the heating power scan (e.g. you might decrease electron cyclotron resonance heating (ECRH) in 0.5 MW steps). Check that the DR (and any other fluctuation measurements) will still be able to make quality measurements in the radial region of interest during the ECRH scan.

Step 4: Set-up an input file for gyrokinetic simulations of the target discharge. Run linear gyrokinetic simulations on the target 'base case' (experimental inputs used with no changes) and perform sensitivity scans to understand what mode or modes are unstable in the radial regions of interest. This step is critical to identify the gyrokinetic model to use in the nonlinear gyrokinetic simulations in the next step (electromagnetic or electrostatic, ion-scale versus electron-scale turbulence, drift kinetic versus gyrokinetic species, impurities, etc.).

Step 5: If resources permit, run nonlinear simulations of the target plasma as well. Run wavenumber and/or radial resolution scans, box-size scans, etc. to develop an understanding of how to get a numerically robust result for these plasma conditions. Take special care to numerically resolve the linear instability identified from Step 4. Most actual experimental conditions live very close to marginal stability, and the simulations are much more challenging and computationally intensive to run compared to more theoretical cases like the cyclone base case. If resources permit, change the input gradients and other input parameters in a way that mimics how the actual plasma parameters might change during the ECRH scan. If resources permit, attempt to predict what changes would be seen in the turbulence data using a synthetic diagnostic $(\S 5)$.

Step 6: Run the actual experiment and analyse the data. Iterate the data analysis steps $(\S \S 3.1-3.3)$ as needed to generate kinetic equilibrium reconstruction, smooth profile fits and well-constrained power balance calculations. Rigorous analysis techniques exist to aid in quantifying the uncertainties on the experimental data sets, as discussed in $\S 3$.

Step 7: Set-up an input file for gyrokinetic simulations of the new experimental discharge. Run linear gyrokinetic simulations on the 'base case' and perform 
sensitivity scans to understand what mode or modes are unstable in the radial regions of interest. If nonlinear simulations have not yet been run in step 5, then first perform resolution scans, box-size scans, etc. so that you can develop an understanding of how to get a numerically robust result.

Step 8: Run nonlinear simulations on the 'base case' and compare with power balance results. A good approach is to perform sensitivity scans of input parameters within error bars to obtain a 'flux-matched case', when using gradient-driven simulations. It is an important result if the simulation can (or cannot) match the experiment ion and electron heat fluxes from power balance within error bars. If we want to apply a synthetic diagnostic to output data from the nonlinear simulation, the simulations need to adequately resolve the turbulence wavenumber detected by the DR system in this example. This may require a higher resolution simulation than is required to study heat fluxes. In addition, application of a synthetic diagnostic (Step 9) may require the generation of synthetic time history, with sufficient temporal resolution to allow for application of the same data analysis routines used on experimental time histories.

Step 9: Once a 'flux-matched case' is obtained, apply the synthetic diagnostic for the DR and compare with the measured turbulence characteristics. Perform sensitivity scans of synthetic diagnostic parameters (e.g. the wavenumber resolution can be scanned and/or the spot size, or sample volume size, can be scanned within experimental uncertainty). If possible, go back and scan the inputs to the gyrokinetic code and apply the synthetic diagnostic for the DR, to gauge how sensitive the turbulence is to changes in the input parameters. The sensitivity may be different than that of the fluxes.

Step 10: Apply a validation metric using as many of the measurements as possible to better quantify and interpret the meaning of agreement and/or disagreement between the experiment and the code. Practical examples can be found in the following references: Ricci et al. (2011), Holland (2016), Freethy et al. (2018).

Step 11 and so on: Repeat steps above, as needed, to explore robustness of agreement and disagreement found between experiment and simulation.

\section{Data analysis in support of gyrokinetic code validation}

Designing dedicated experiments with the goal of gathering a data set suitable for extensive gyrokinetic code validation is important because a single, rich data set can be a good foundation for a variety validation studies, and can be compared with many different models and codes. This section will describe the basic building blocks of data analysis used for validation experiments in fusion plasmas: magnetic equilibrium reconstruction, $\S 3.1$, radial profile measurements, $\S 3.2$ and power balance calculations, $\S 3.3$, as well as discussing preparation of input files for gyrokinetic codes, $\S 3.4$.

\subsection{Magnetic equilibrium reconstruction}

For magnetic equilibrium reconstruction (Mukhovatov \& Shafranov 1971), a variety of experimental data can be used. Most routinely, an equilibrium reconstruction is obtained using data only from magnetic diagnostics (magnetic probes, flux loops, coil currents, diamagnetic flux, etc.) to constrain the solution of the Grad-Shafranov equation. A variety of codes in the experimental community are used routinely for this purpose, two examples being CLISTE (McCarthy, Martin \& Schneider 1999) and EFIT (Lao et al. 1990). To test that the magnetic reconstruction does not contain any systematic uncertainties, a variety of consistency checks are possible. One very simple 

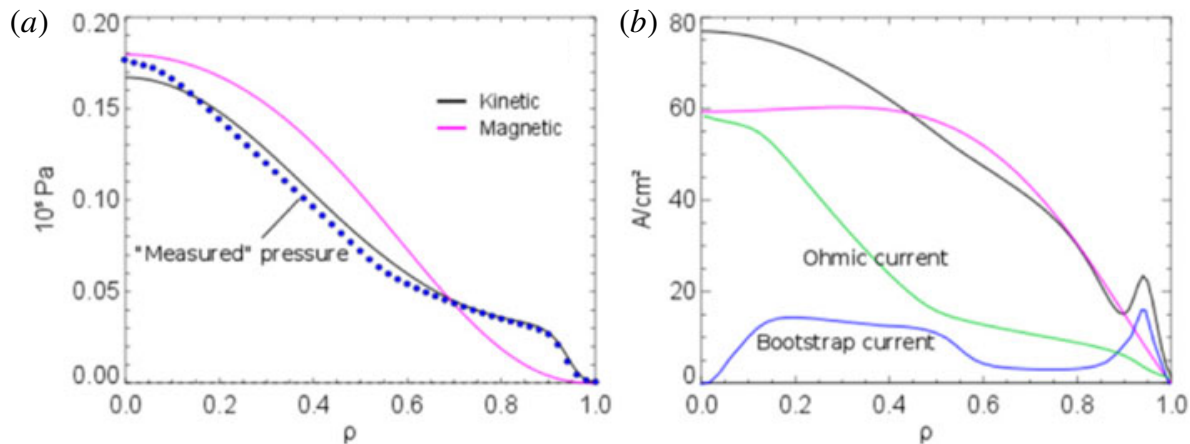

(c)

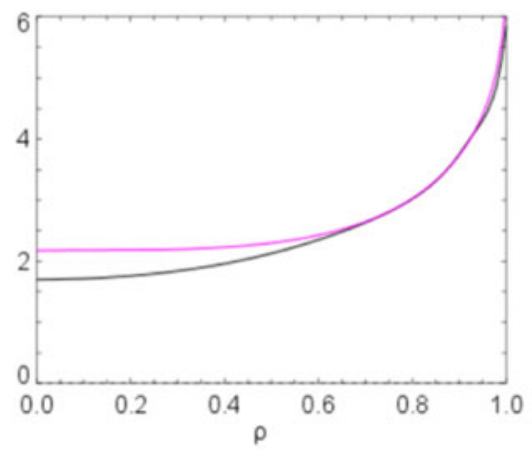

(d)

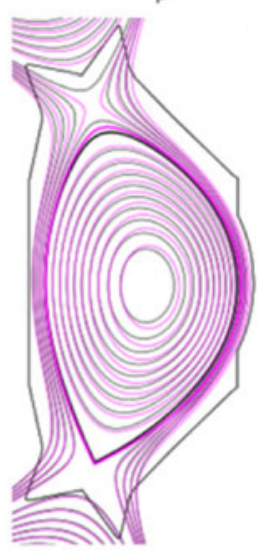

FIGURE 3. Kinetic equilibrium reconstruction (black) compared to a magnetics only reconstruction (pink). Reproduction of figure 2 of Li et al. (2013).

method involves comparing high-field side (HFS) electron cyclotron emission (ECE) measurements to low-field side (LFS) ECE measurements. Since electron temperature, $T_{e}$, is expected to be a constant on a flux surface, if the ECE measurements mapped onto the reconstruction from HFS and LFS disagree, then one should suspect that the position of the magnetic axis (according to the reconstruction) may be incorrect.

The extension of this is the 'kinetic equilibrium reconstruction', where pressure profile measurements in addition to magnetic measurements are used to constrain the reconstruction. An example of the difference between a 'magnetics only equilibrium reconstruction' and a 'kinetic equilibrium reconstruction' is shown in figure 3 . There can be significant differences in the safety factor profile and flux surface location in real space in tokamak plasmas featuring strong auxiliary heating and current drive.

In addition to kinetically constrained equilibrium reconstructions, a typical goal of dedicated validation experiments is to obtain measurements of the plasma current profile, from the motional Stark effect (MSE) (Holcomb et al. 2008) or polarimeter (Bergerson et al. 2012) measurements. Using more data to constrain the magnetic equilibrium reconstruction is useful for addressing both systematic and random uncertainties. Reducing the uncertainty on the equilibrium reconstruction will help reduce the errors in diagnostic mapping, that is, determining where a measurement made in real space maps to in flux geometry.

Using more data to constrain a magnetic reconstruction means that any errors or uncertainties on that data will propagate though to the final result. It is desirable 

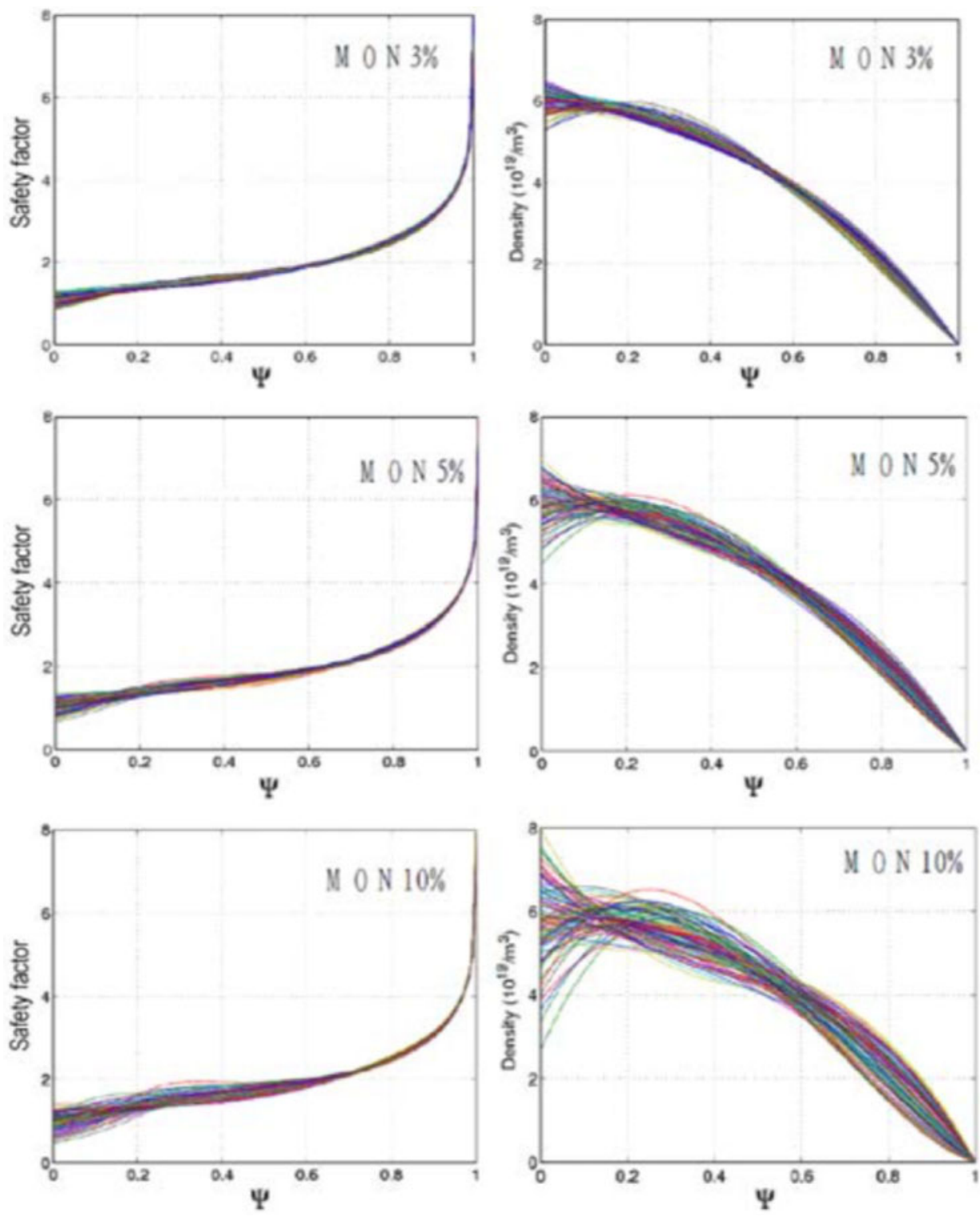

FIGURE 4. Error bars on the safety factor $q$ arising from random error in magnetics data at the EAST tokamak are visualized by overplotting the $q$ and density profiles reconstructed with $3 \%, 5 \%$ and $10 \%$ random perturbations from the original equilibrium. Reproduced from figure 5 of Qian et al. (2015).

that errors on safety factor and magnetic shear be as low as possible. Random error on these reconstructed values (and on any output value from the reconstruction) can be estimated using a simple Monte Carlo approach, by varying the inputs all randomly within the error bars, and running the reconstruction code many times to obtain mean output values and variances on the mean. One example of results from this approach is shown in figure 4. Other, faster methods, such as semi-analytic approaches to estimate the uncertainty in the equilibrium free parameters can be 
employed (Anderson et al. 2004), and open-source toolsets for quantifying the error and uncertainty on the magnetic equilibrium reconstruction are available (Chilenski, Faust \& Walk 2017).

\subsection{Radial profile measurements}

Reliable techniques to measure electron density and electron temperature are widely available, exploiting Thomson scattering (measurements of $n e(r, t), T e(r, t)$ ) and ECE diagnostics $(T e(r, t))$, and profile reflectometers (ne $(r, t))$ (Donne 2007). Thomson scattering density profile measurements can be additionally constrained using data from multi-chord interferometers or other measurements, or data from profile reflectometers. Ion temperature and rotation measurements are far more challenging, typically requiring beam based charge exchange (Fonck et al. 1984), e.g. with carbon (Grierson et al. 2012) or boron and nitrogen (Viezzer et al. 2012), or X-ray imaging crystal spectroscopy using argon (Reinke et al. 2012). Note that in these cases the measured ion temperature and rotation are those of the measured impurity, which in many cases may be argued to have the same values as for the main ions (Hutchinson 1987). Specialized measurements and dedicated experiments are required to extract the main ion temperature and rotation profile data directly (Grierson et al. 2012). Care must also be taken to constrain estimates of the impurity content, or $Z_{\text {eff }}$ profile, in the plasma. This discussion of profiles is limited to one-dimensional (1-D) radial profiles, and any poloidal asymmetries of the temperature or density are ignored, which is an acceptable approximation in the core of the plasma, where the standard gyrokinetic theory is valid. It is important to note this approximation fails in many cases for the impurity density or plasma rotation.

The experimental value of local temperature (or density, rotation, etc.) and the local radial gradients are derived from smooth fits to the 1-D radial profiles of the data. In figure 5, data from an Alcator C-Mod ICRF heated L-mode plasma from a dedicated experiment are shown, along with fits to the data using several different techniques. The use of integrated data analysis (IDA) procedures and the Bayesian approach is extremely beneficial to constrain the reported 'fits' to experimental measurements (Fischer \& Dinklage 2007; Rathgeber et al. 2010; Reusch et al. 2014). Gaussian process regression (GPR) can be used to fit the profiles and calculate the error bars (Chilenski et al. 2015). Simpler methods, such as spline fits combined with a Monte Carlo approach have also been used in the past (White et al. 2010b; Holland 2016). With any profile fitting method, it is not uncommon to end up with uncertainties on normalized gradient scale lengths, e.g. $a / L_{\mathrm{ne}}, a / L_{\mathrm{Te}}$ and $a / L_{\mathrm{Ti}}$, between 10 and $30 \%$.

All profile measurements are not required on every shot in a dedicated experiment; in many experiments it is sufficient to run a reference shot (to obtain the desired profile and/or turbulence measurements), and then run repeat shots to obtain all the other data of interest. Of course, the reference and repeat discharges must have matched parameters within experimental error bars. The data from the reference shot (or averaged data from several reference shots or several time slices within a reference shot) are used for gyrokinetic simulation.

\subsection{Power balance calculations}

Minimizing the errors and uncertainty of the simulation inputs taken from the magnetic equilibrium and the measured radial pressure profiles is just one step in experimental design and execution. Because experimental fluxes can only be directly measured in very specialized experiments using diagnostics that are not widely 
(a)

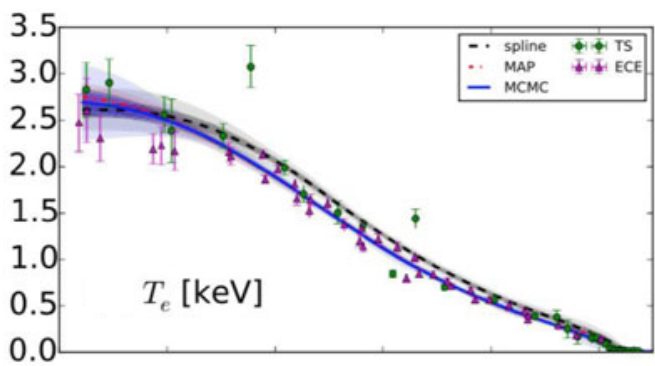

(b)

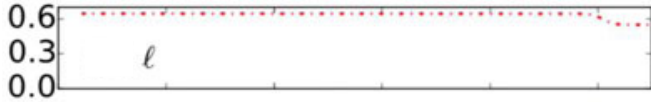

(c)

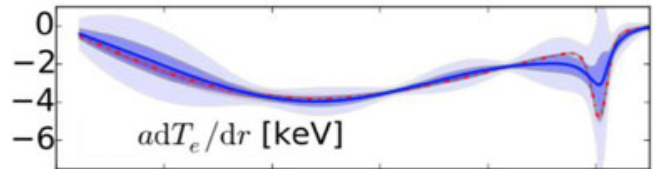

$(d)$

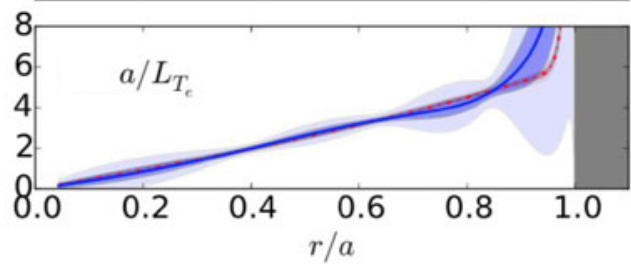

FIGURE 5. Gaussian process regression (GPR) methods are used to fit electron temperature data in C-Mod, and rigorously quantify the error bars that will constrain the later sensitivity scans in a validation study. From figure 6 of Chilenski et al. (2015).

available (Forster et al. 1994; Evensen et al. 1998; Howard et al. 2012), power balance calculations are used to infer the experimental values of the heat and particle fluxes. It is very important to minimize the error on the experimental values fluxes calculated by power balance for gyrokinetic validation studies.

A power balance calculation solves the electron and ion energy conservation equations, the poloidal magnetic diffusion equation and the particle and momentum balance equations. Additional models are employed to handle neutral transport, plasma conductivity and auxiliary heating and current drive. Reducing error bars on interpretive power balance calculations is complicated by the need to use many measurements as input to the power balance calculation. For example, to solve for the electron and ion heat fluxes, power balance calculations will take as input the profiles we have already discussed, $T e(r, t), T i(r, t), n e(r, t)$ and also will need radiated power profile measurements, current profile and information about $Z_{\text {eff }}$. The power balance codes also use the total heating power and other characteristics of specific actuators (e.g. ion cyclotron range of frequency (ICRF) heating or electron cyclotron resonance heating $(\mathrm{ECRH})$ as input). The profile of energy deposition is then calculated self-consistently using models such as TORIC (Brambilla 1999).

The primary equation solved in power balance is the electron energy conservation equation (Ongena et al. 2012):

$$
\frac{3}{2} \frac{\partial}{\partial t}\left(n_{\mathrm{e}} T_{\mathrm{e}}\right)+\nabla \cdot \boldsymbol{q}_{\mathrm{e}}+\nabla \cdot\left(\frac{5}{2} n_{\mathrm{e}} T_{\mathrm{e}} \boldsymbol{v}_{\mathrm{e}}\right)+\boldsymbol{v}_{\mathrm{i}} \cdot \nabla\left(n_{\mathrm{i}} T_{\mathrm{i}}\right)=S .
$$


The electron heat flux is $\boldsymbol{q}_{\mathrm{e}}=n_{\mathrm{e}} \boldsymbol{\nabla} T_{\mathrm{e}} \chi_{\mathrm{e}}$, and the thermal diffusivity, $\chi_{\mathrm{e}}$, is equal to the electron thermal conductivity divided by electron density. As described in Ongena et al. (2012) the first term in the electron energy conservation equation above is the rate of change of the electron energy, the second term gives the heat flux, the third is the electron convection losses (arising from the particle flux) and the fourth is work done by the plasma particles against the pressure gradient. Terms on the right-hand side, $S$, are the power gain and loss terms for the electrons, such as ohmic heating power, auxiliary power to electrons (ECRH, ICRF, etc.), radiation losses, ionization losses and exchange losses to ions. The electron energy conservation equation is solved for the unknown thermal diffusivity (for the unknown heat flux in term two on the left-hand side) using experimental measurements and models to assess the other terms. A similar energy conservation equation is solved for the ions to obtain the unknown ion heat flux.

In practice, codes like TRANSP (Hawryluk 1979) and ASTRA (Pereverzev et al. 1991) are commonly run after an experiment in 'interpretive mode', using experimental kinetic profiles as input, along with appropriate models for the power gain and loss terms. This is in contrast to running power balance in predictive mode, for which kinetic profiles are predicted using transport models (Budny et al. 2008). Once an interpretive power balance run is complete, several internal consistency checks are possible to reveal systematic errors in power balance calculations. The measured neutron rate and loop voltages can be compared to modelled values. The stored energy can be calculated and compared to that of the magnetic reconstruction (which is also a step that is part of assessing the fast ion pressure contribution to the total ion pressure). Systematic uncertainties can be identified via these and other methods (Petty et al. 1998; Grierson et al. 2018). For an excellent pedagogical review of power balance calculations see Ongena et al. (2012) and for more rigorous derivation of transport equations see Hinton \& Hazeltine (1976). As an additional point of interest, perturbative transport experiments are also used to calculate an experimental 'heat pulse' or 'perturbative diffusivity'. This is not to be confused with the 'steady state' or equilibrium diffusivity solved for by power balance. Recent examples of using the perturbative diffusivity to compare with turbulent transport models can be found in Smith et al. (2015), Creely et al. (2017) and references therein.

Error analysis of interpretive power balance runs is non-trivial, and several different approaches have been discussed in the literature, along with identification of common systematic errors (Holland 2016). Analytic error propagation gives good agreement with Monte Carlo approaches (White et al. 2010b). More sophisticated methods for calculating the errors on power balance results have been recently proposed (Vaezi \& Holland 2018). Despite best efforts, the experimental error can be reduced only so much, and the ion and electron heat fluxes, $Q_{\mathrm{i}}$ and $Q_{\mathrm{e}}$, will typically have errors of $\sigma_{Q} \sim 10-30 \%$. Shown in figure 6 is an example of ion and electron heat fluxes that were experimentally inferred at DIII-D using power balance in a targeted validation experiment (Holland 2016), which illustrates the radial variation of the fluxes and uncertainties.

Once the power balance analysis is complete and verified to be free of systematic errors (Holland 2016), it is important to take care when selecting which outputs correspond to the 'experimental values' to be used for comparison with the gyrokinetic codes. For example, a gyrokinetic transport model will calculate the heat flux due to the turbulence. Typically, power balance codes give the convection losses arising from the particle flux as a separate contribution to the total heat flux 

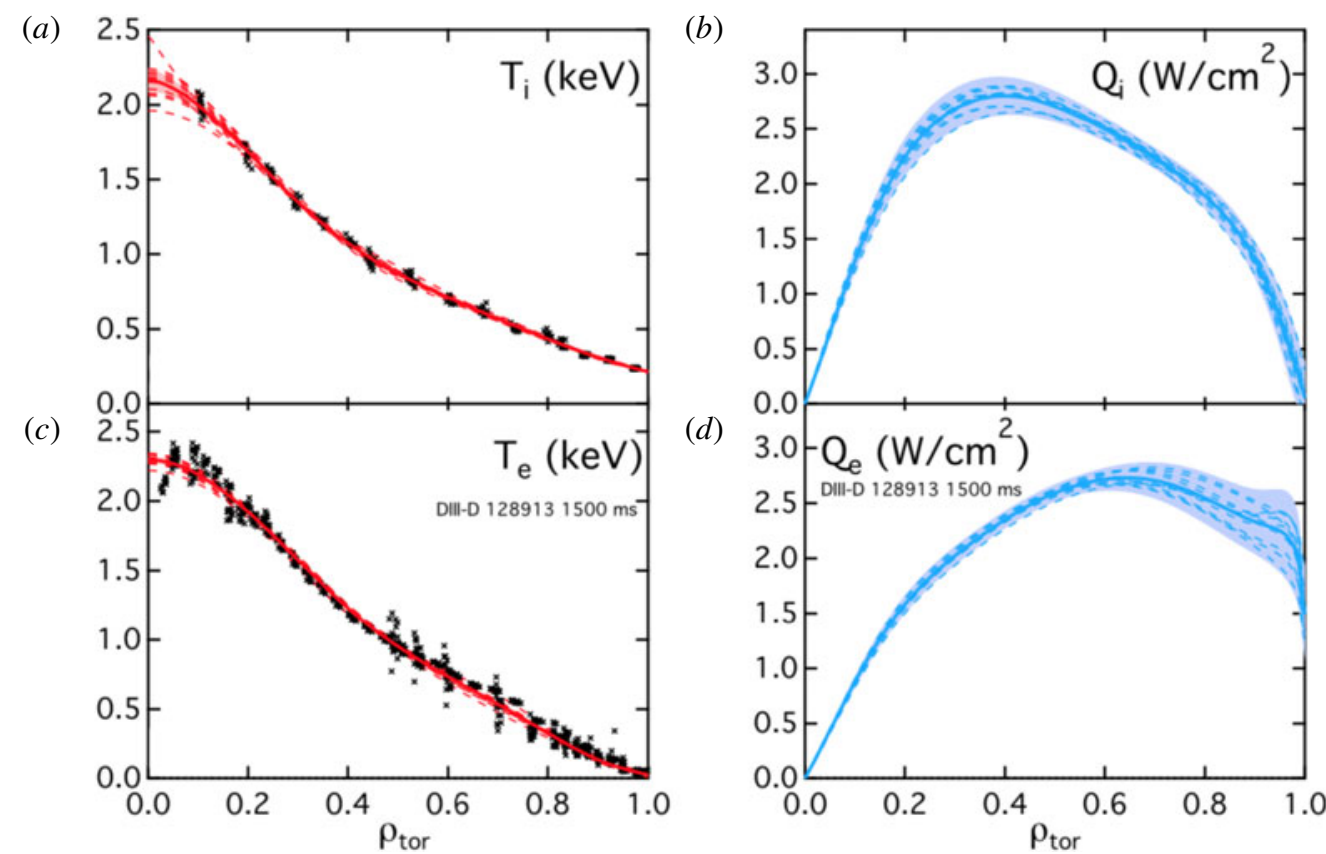

FIGURE 6. The errors on temperature profiles are propagated through the power balance calculation to obtain errors on the inferred ion and electron heat fluxes. From figure 6 of Holland (2016).

(Ongena et al. 2012). The convection losses are small compared to conducted losses, but may not be negligible. For example then, when we run transport models to calculate turbulent and neoclassical fluxes, we get a total flux, which should be compared to the sum of the 'heat flux loss' and 'convection loss' terms in the electron energy conservation equation solved by TRANSP (Ongena et al. 2012); though again, the convection loss term is usually very small.

A significant systematic error can arise from handling the fast ion contribution to the ion pressure. If the fast ion pressure is substantial, then this must be modelled within power balance correctly (e.g. using additional modules that calculate the fast ion transport due to collisions). For discussion of the fast ion transport power balance calculation process and associated uncertainties, see Pace et al. (2013). Integrated modelling approaches are also very useful in plasmas with substantial fast ion pressures, as described in Garcia et al. (2015), where differences between the fast ion pressure and the thermal ion pressure can be significant. In addition to ensuring correct interpretation of power balance results, assessment of the fast ion pressure is important in plasmas where the superthermal pressure gradients has direct impact on the turbulence being simulated by gyrokinetic codes (Citrin et al. 2014).

\subsection{Preparing input files for gyrokinetic codes}

To prepare an input file a flux-driven gyrokinetic simulation of an experimental discharge requires at minimum the experimental equilibrium reconstruction and local pressure profile measurements. Using the experimental data, suitably processed, input files for the gyrokinetic code are prepared for each code using specialized tools. 
Sometimes the specialized tools use outputs from a power balance code, in which case a power balance analysis will also be required before the input files for the simulation can be prepared. Obviously the details of how to prepare an input file for a gyrokinetic code will depend on the specific experiment as well as the code of interest, but there are several general issues that are useful to keep in mind. One pitfall has to do with definitions of fundamental parameters of interest. For example, the measured values of temperature and local magnetic field (based on an equilibrium reconstruction) may be used by an experimentalist to calculate the value of the ion sound gyroradius $\rho_{\mathrm{s}}=\left(m_{\mathrm{i}} T_{\mathrm{e}, \text { local }} / 2 B_{\text {local }}\right)^{1 / 2}$ at a particular radiation location in an experiment. However, within the native units of a gyrokinetic code, the value of $\rho_{\mathrm{s}}$ may be very different at the same radial position. This should be carefully tracked when comparing the reference magnetic fields used in local, flux-tube simulations with values obtained from experiments. Miller geometry is often used to parameterize the magnetic equilibrium (Miller et al. 1998), which can also affect the mapping from experimental coordinates to simulation coordinates. Finally, we note that care must be taken to ensure that the local gradient of the profiles is consistently calculated between the experiment and the codes. It is good practice (part of identification and quantification of uncertainty (Terry et al. 2008)) for authors to specify the simulation numerical details, as well as the input parameters and definitions, in validation papers.

\section{Turbulence measurements in the core of fusion plasmas}

At most major fusion devices around the world there are several fluctuation diagnostics that can be used in validation studies. Density fluctuation measurements are the most widely available. Beam emission spectroscopy (BES) diagnostics (McKee et al. 2007; Field et al. 2014), and standard reflectometry (Nazikian, Kramer \& Valeo 2001) are used to probe long-wavelength or low- $k$ turbulence (typically $k_{\theta} \rho_{\mathrm{s}}<0.5$ ), relevant for ITG/TEM type turbulence or MTM type turbulence. In the intermediate wavelength range, Doppler reflectometer (also known as Doppler backscattering) (Holzhauer et al. 1998; Hirsch et al. 2001; Conway et al. 2004; Hennequin et al. 2004; Happel et al. 2009; Hillesheim et al. 2009; Rhodes et al. 2010; Tokuszawa et al. 2012; Zhou et al. 2013) can access wavenumbers between the high ion scale $k_{\theta} \rho_{\mathrm{s}} \sim 0.5$ and the low- $k$ electron scale, $k_{\theta} \rho_{\mathrm{s}} \sim 5$. At the highest wavenumbers, relevant to ETG-scale turbulence, density fluctuations can be measured with high $k$ coherent scattering diagnostics (Rhodes et al. 2007; Smith et al. 2008; Qing et al. 2010).

Electron temperature fluctuations can currently only be accessed at long-wavelength or low $k$, with correlation electron cyclotron emission (CECE) diagnostics (Sattler \& Hartfuss 1993, 1994; Cima et al. 1995; White et al. 2008; Zemedkun et al. 2015; Freethy et al. 2016; Sung et al. 2016; Fontana, Porte \& Cabrera 2017) on tokamaks and stellarators; unfortunately this measurement is unavailable in spherical tokamaks, because the 2 nd harmonic $\mathrm{X}$-mode electron cyclotron emission is cutoff in these low toroidal field, high plasma beta plasmas. At low- $k$, it is also possible to measure the phase angle between electron temperature and density fluctuations, by coupling an ECE radiometer with a reflectometer (White et al. 2010a).

Turbulence fluxes cannot be measured directly with any of these systems. Systems like the heavy-ion beam probe (Forster et al. 1994) can measure the electrostatic potential fluctuations and density fluctuations simultaneously, and hence the turbulent particle flux, but these measurements have not been fielded widely.

The review article of Tynan et al. (2009) includes an extensive discussion of turbulence measurements in fusion devices and basic plasma devices. In this tutorial, 
we discuss in detail only a handful of fluctuation measurements: beam-emission spectroscopy diagnostics (BES), correlation electron cyclotron emission (CECE) radiometers, $n-T$ phase diagnostics, Doppler reflectometers and coherent scattering measurements. These measurements are discussed in order to highlight general turbulence data analysis techniques used in validation studies.

Additional turbulence diagnostics used to measure core turbulence in fusion devices, some of which have been compared to gyrokinetic simulation, but not discussed in this tutorial, include: heavy ion beam probes (Forster et al. 1994), fast profile reflectometer (Casati et al. 2009), phase contrast imaging (PCI) (Ennever 2016), fast two colour interferometry (FTCI) (Kasten, White \& Irby 2014), polarimeter (Bergerson et al. 2012) cross-polarization scattering (Rhodes et al. 2014), ultra-fast charge exchange recombination spectroscopy (UF CHERS) (Evensen et al. 1998; Uzun-Kaymak, Fonck $\&$ McKee 2012).

\subsection{Beam-emission spectroscopy diagnostic}

Beam-emission spectroscopy (BES) systems are used to measure local density fluctuations from collisionally excited, Doppler-shifted emission from neutral beams, usually hydrogen or deuterium (McKee et al. 2007; Tynan et al. 2009). The fast neutral atoms injected by the beams are excited via plasma collisions. The excited neutrals emit $\mathrm{H}$-alpha or D-alpha photons due to collisional and radiative processes. These photons are blue shifted due to the beam velocity, which allows a separation from other $\mathrm{H}$-alpha or D-alpha emission. The normalized fluctuation intensity of the emission is related to the normalized density fluctuation using a collisional radiative model (Hutchinson 2002). BES is a useful measurement for validation because it is highly localized in radius, and has good time resolution intrinsically, though when measuring small fluctuation levels, time averaging over steady phases of the discharge is required. Unlike reflectometry, measurement of an absolute fluctuation level $\tilde{n} / n$ rather than in arbitrary units (a.u.) is possible with atomic physics modelling. However, neutral beam injection is required, which may be perturbative or unavailable in the plasmas of interest.

With BES or any other fluctuation diagnostic, the frequency spectrum is measured in the laboratory frame, as shown in figure 7. The spectral shape is determined by the sum of the fluctuation frequency in the plasma frame and the Doppler shift arising from the radial electric field. When the Doppler shift is dominant, the measured frequency is roughly proportional to the poloidal wavenumber of the fluctuations. (McKee et al. 2000).

By integrating the autospectrum over a given frequency range of interest, with appropriate normalization, the fluctuation level is calculated. Note that if the entire frequency range were to be used, the fluctuation level recovered should be identical to the root-mean-square (r.m.s.) fluctuation level calculated from the time history data (Pareseval's theorem). Performing measurements at different radii allows the profile of the fluctuation levels to be reconstructed, as shown in figure 8. If it is not possible to obtain all the profile data points in one reference discharge, several repeat discharges are used, as long as the plasmas are identical within the experimental error bars (White et al. 2008).

The measured fluctuation spectrum will be perturbed by the presence of Magnetohydrodynamic (MHD) mode activity, and the total fluctuation level measured will reflect the effects of drift-wave turbulence combined with MHD (Field et al. 2014). It is important in Gyrokinetic (GK) validation experiments to design discharges 


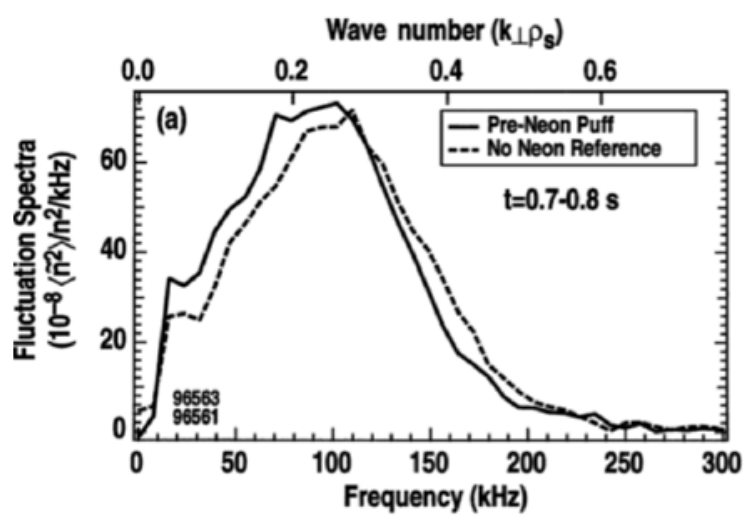

FIGURE 7. BES measurements of turbulent density fluctuations in two L-mode plasmas in DIII-D are shown. In both cases, the measured turbulent frequency spectrum is mapped to the wavenumber spectrum by exploiting the fact that the Doppler shift dominates over the frequency of the fluctuations in the plasma frame in these cases. From figure 3(a) of McKee et al. (2000).

with little to no MHD activity (e.g. tearing modes and sawteeth) if the effects on turbulence and transport are not included in the transport models.

The correlation length of the turbulence is constructed using pairs of signals at different measurement locations. From the plot of the correlation coefficient at zero time delay versus spatial separation, it is possible to define the correlation length $L_{c}$, as the width of a Gaussian function fitted to the envelope of the time-delay correlation function $C(\tau=0)$ plotted versus the distance $x$ between channels: $C(\tau=0)=\exp \left(-\left(x / L_{c}\right)^{2}\right)$ (Shafer et al. 2012). Other definitions may be used; it is best to verify with the diagnostician how the correlation length parameter is being calculated. It is also important to note that there can be limitations when using the two-point correlation technique method (Kim et al. 2016). With 2-D imaging BES systems, it is possible to measure radial and poloidal correlation lengths, and even to extract detailed information about the eddy sizes, shapes, and tilts, from measured 2-D correlation functions. These parameters have been compared with gyrokinetic simulations (Shafer et al. 2012).

\subsection{Correlation electron cyclotron emission (CECE) measurements of ion-scale electron temperature fluctuation measurements}

Correlation electron cyclotron emission (CECE) measurements exploit well understood diagnostic principles of detecting electron cyclotron emission from the optically thick second harmonic X-mode electron cyclotron resonance layer in fusion plasmas (Hutchinson 1987). Radiometers are commonly used to measure the radial profile of electron temperature from ECE in fusion plasmas with excellent spatial and temporal resolution. A radiometer measures the temperature of a radiating thermal source by applying a band-pass filter to the incoming white noise signal. This means that a single radiometer channel suffers from a baseline noise level, called the thermal noise level. The thermal noise level is set by the bandwidths of the pre-detection filter and post-detection filter (Cima et al. 1995). A standard multi-channel radiometer is optimized for making radial profile measurements of the electron temperature. 

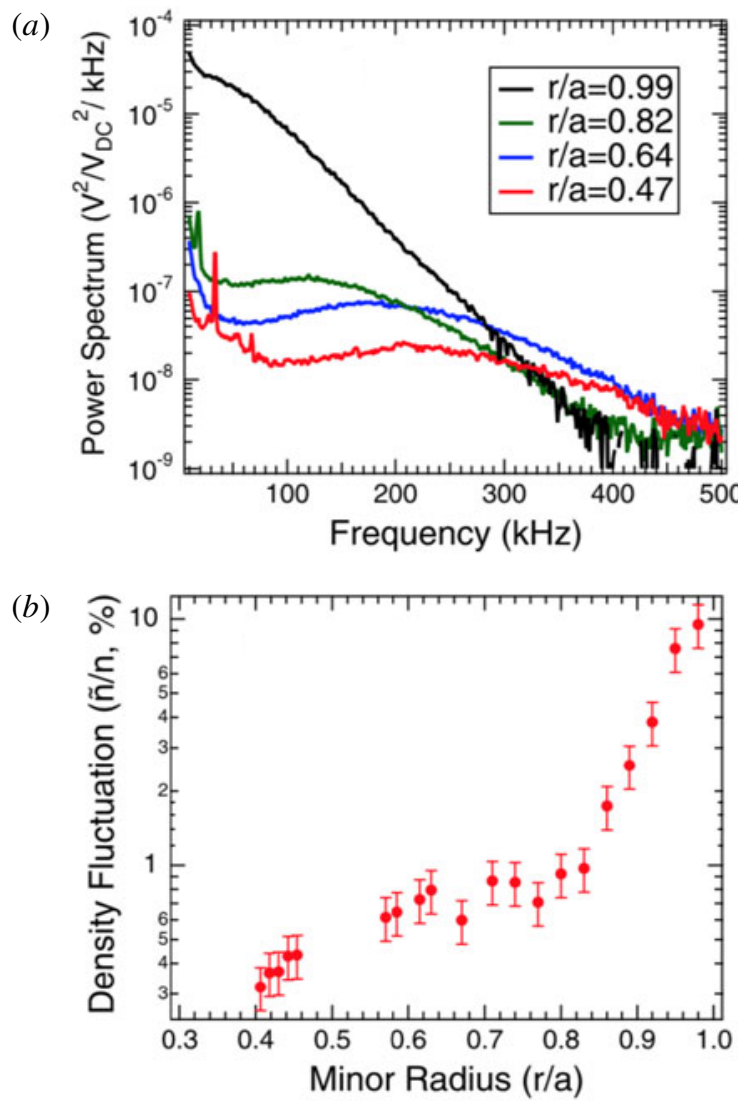

FIGURE 8. Radial profile of fluctuation spectra measured with BES in DIII-D in an Lmode plasma. The spectra are integrated over the frequency range of interest to calculate the reported fluctuation level $\tilde{n} / n$. From figure 3 of McKee et al. (2007).

This means that the collection optics are not deigned allow for a tight focus in the plasma. It also means that the receiver electronics will have rather narrowband post-detection filters (setting the frequency response of the system to low values, in the $10 \mathrm{~s}$ of $\mathrm{kHz}$ range) and fairly wideband pre-detection filters (setting the radial resolution to be comparable to the ECE linewidth, approximately $1 \mathrm{~cm}$ or so in typical medium-scale fusion devices). Dedicated radiometers for fluctuation measurements are designed with focusing optics that achieve a sample volume comparable to the turbulent length scales of interest. The receiver will have pairs of channels with wideband post-detection filters (setting the frequency response of the system to high values in the $\mathrm{MHz}$ range) and with very narrow-band pre-detection filters (setting the radial resolution to sub-cm scale in typical medium-scale fusion devices). This latter choice is made so that two measurement locations can be co-located within the radial correlation length of the turbulence without having the bandwidths overlap in frequency, which ensures that the thermal noise is not correlated between the two channels.

The CECE measurement relies on correlating two such radiometer channels with the same turbulent signals, but with different thermal noise signals (Sattler \& Hartfuss 1993; Cima et al. 1995), and has been most widely deployed using a frequency-based 


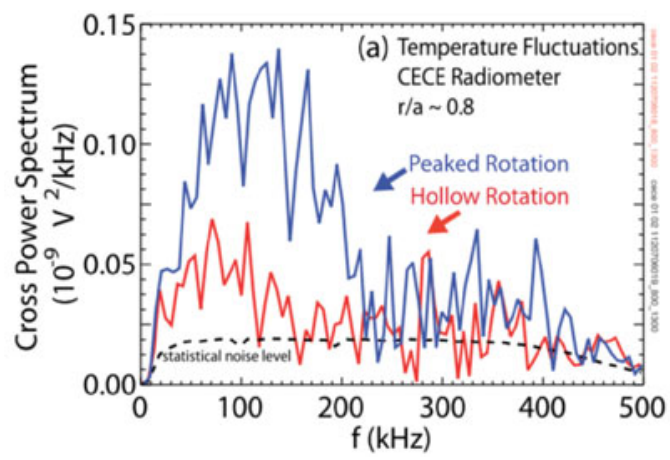

FIGURE 9. The spectrum of turbulent electron temperature fluctuations is measured in two L-mode plasmas with different intrinsic rotation profiles in Alcator C-Mod. The spectra are integrated over a frequency range of interest to calculate fluctuation levels. From figure 6(a) of White et al. (2013).

decorrelation method (White et al. 2008; Zemedkun et al. 2015; Freethy et al. 2016; Sung et al. 2016). Correlation ECE radiometers have good spatial resolution, and can provide quantitative fluctuation levels in very straightforward manner, and are non-perturbative and passive measurements. Time averaging ranging from $50 \mathrm{~ms}$ to $1 \mathrm{~s}$ depending on the details of the turbulence is required to reduce thermal noise and extract the turbulent fluctuation data (White et al. 2008). In addition, plasma density and magnetic field values limit accessibility, as with other mm-wave diagnostics like reflectometry and Doppler reflectometry. CECE radiometers tend to be most sensitive to low- $k$, ion-scale, electron temperature fluctuations $\left(k_{\theta} \rho_{\mathrm{s}}<0.5\right)$.

Care must be taken with CECE measurements to fully exclude contributions of the thermal noise to the reported fluctuation level when calculating the cross-power spectrum between two radiometer channels. The resulting CECE cross-power spectrum can then be interpreted as the frequency spectrum of the electron temperature fluctuations. Figure 9 shows an example of CECE data from the C-Mod tokamak, where L-mode plasmas featuring different radial profiles of intrinsic rotation (White et al. 2013) are found to have different fluctuation levels. Integrating under the measured cross-power spectrum gives the fluctuation level, similar to analysis performed with BES data. Making this measurement at different radial locations with a multi-channel system, the CECE fluctuation amplitude radial profile can be measured. Figure 10 shows an example from TCV. The temperature fluctuation radial profiles are shown for two different triangularity plasmas (Fontana et al. 2017), which are found to have different fluctuation levels (again, corresponding to different transport characteristics). The radial and poloidal correlation lengths of the electron temperature fluctuations have also been measured in a number of experiments using standard 1-D ECE radiometers (Freethy et al. 2018) and 2-D imaging ECE radiometers (Deng et al. 1998).

\subsection{The $n-T$-phase measurements}

Coupled reflectometer and radiometer (ECE) systems can be used to measure the cross-phase angle between low- $k n_{\mathrm{e}}$ and $T_{\mathrm{e}}$ fluctuations in stellarators and tokamaks (Haese et al. 1999; White et al. 2010a; Hillesheim et al. 2013; Cao et al. 2016; Freethy et al. 2016). An example of the measurement in an ohmic L-mode plasma 


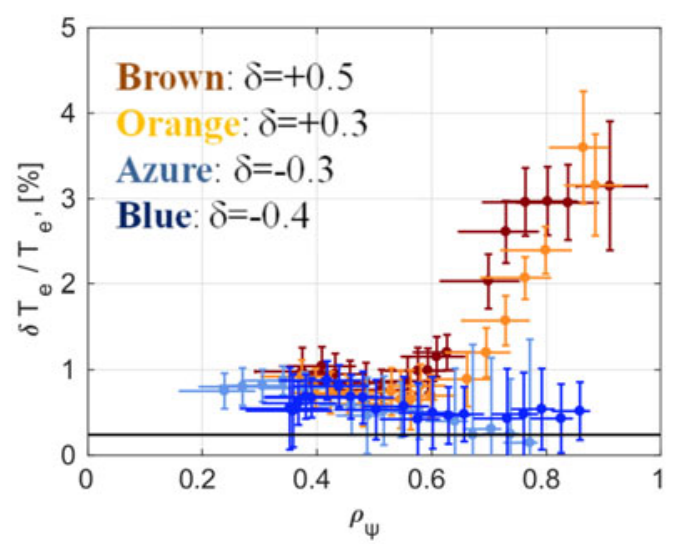

FIGURE 10. Radial profiles of the relative electron temperature fluctuation amplitude in plasmas with different triangularity values in TCV, as measured with a correlation ECE radiometer. From figure 5 of Fontana et al. (2017).

at DIII-D is shown in figure 11. The wavenumber resolution is determined by the spot-size for the ECE radiometer optics, and the wavenumber response of the reflectometer.

The cross-phase angle between electron density and temperature (the $n-T$-phase) does not enter directly into the turbulence-driven flux equations, but the measurement is still extremely valuable because it allows for comparisons at multiple levels of the primacy hierarchy. Nonlinear gyrokinetic simulations can be used to calculate the $n-T$-phase angle, allowing for direct comparisons with experimental measurements. Quasilinear transport models can also be used to calculate the dominant (or subdominant) mode's cross-phase angle, which means that measurements of the phase angle can be compared against reduced, theory-based transport models (Bourdelle et al. 2016; Staebler et al. 2016) as well as full nonlinear gyrokinetic models. Linear phase angles tend to track nonlinear phase angles rather well, which makes the phase angle an interesting and robust quantity for comparisons with turbulent-transport models (Dannert \& Jenko 2005; Waltz, Casati \& Staebler 2009; Told et al. 2013). Comparisons of this measurement with simulations may provide information about the mix of ITG and TEM instabilities in the plasma. For example, the sign of the $n-T$-phase angle could be used to identify an instability as an ion branch or electron branch mode (Freethy et al. 2018), similar to measurements of the propagation direction of turbulence in the plasma frame (Horton 1999).

\subsection{Doppler reflectometer (Doppler backscattering) diagnostics}

Turning to a slightly higher $k$ measurement, Doppler reflectometers accesses the intermediate range of wavenumbers, with typical ranges $0.5<k_{\theta} \rho_{\mathrm{s}}<2$ or higher. Doppler reflectometer (DR) (also called Doppler backscattering (DBS)) diagnostics combine the spatial resolution of reflectometry with the wavenumber sensitivity of scattering measurements (Holzhauer et al. 1998; Hirsch et al. 2001; Conway et al. 2004; Hennequin et al. 2004; Happel et al. 2009; Hillesheim et al. 2009; Rhodes 2009; Tokuszawa et al. 2012; Zhou et al. 2013). Doppler reflectometry is an active measurement. A millimetre-wave beam is launched into a plasma at a frequency that approaches a cutoff (O-mode or X-mode) and at an oblique angle 


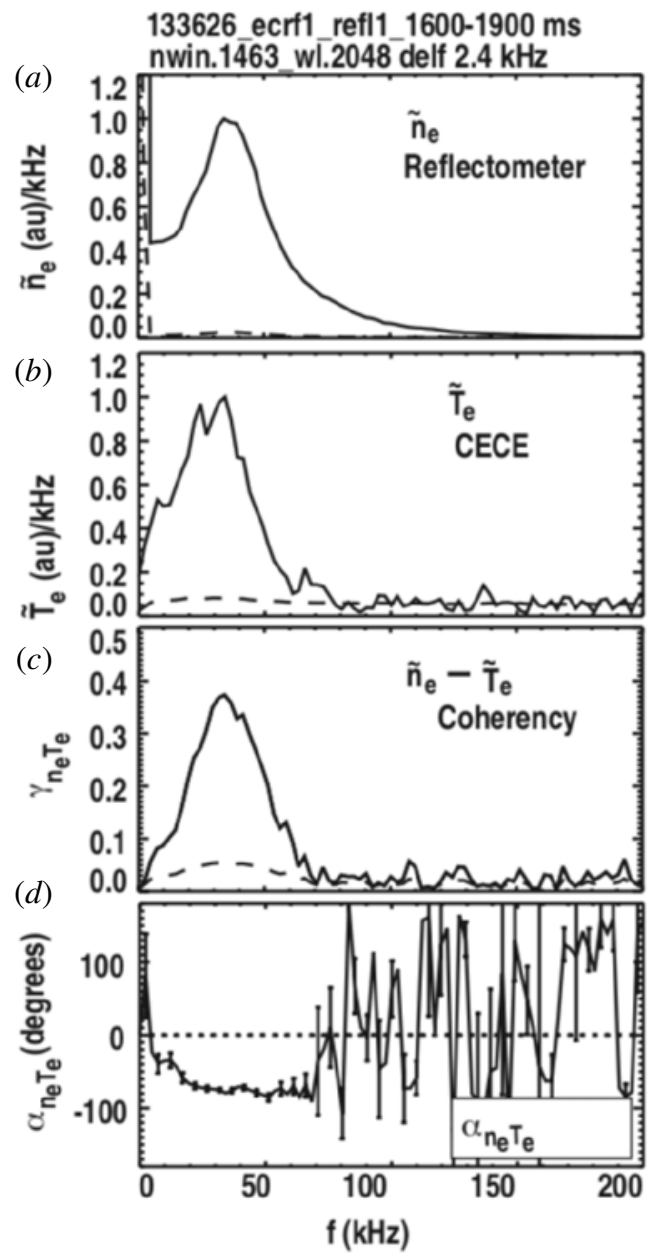

FIGURE 11. Example of data from a reflectometer and CECE radiometer at DIII-D that share the same sight line to the plasma, allowing for measurements of the cross-phase angle between density and electron temperature fluctuations, the $n-T$ phase angle. From figure 3 of White et al. (2010a).

to the cutoff surface. Density fluctuations at particular wavenumber are isolated by choosing frequency of the launched wave, the angle that the launched beam makes with the cutoff surface in the plasmas, and the Bragg scattering. The Bragg condition for $180^{\circ}$ backscattering is given by $k_{\mathrm{o}}=-2 k_{\mathrm{i}}$, where $k_{\mathrm{o}}$ is the wavenumber of the measured density fluctuation and $k_{\mathrm{i}}$ is the incident wavenumber of the probe beam at the scattering location. By varying the angle of the probe beam with respect to the cutoff layer, the wavenumber $k_{\mathrm{o}}$ is varied. A Doppler reflectometer measurement will yield an autopower spectrum, that looks similar to BES or CECE data in appearance. The frequency spectrum from Doppler reflectometers shows a clear peak at a finite frequency. The location of the peak of the spectrum marks the Doppler-shifted frequency, given by $f_{\text {Dopp }}=f_{\text {plasma }}+\boldsymbol{v} \cdot \boldsymbol{k}_{\boldsymbol{o}}$, where $f_{\text {Dopp }}$ is the frequency of the turbulence in the laboratory frame, $f_{\text {plasma }}$ is the frequency of the turbulence in the 


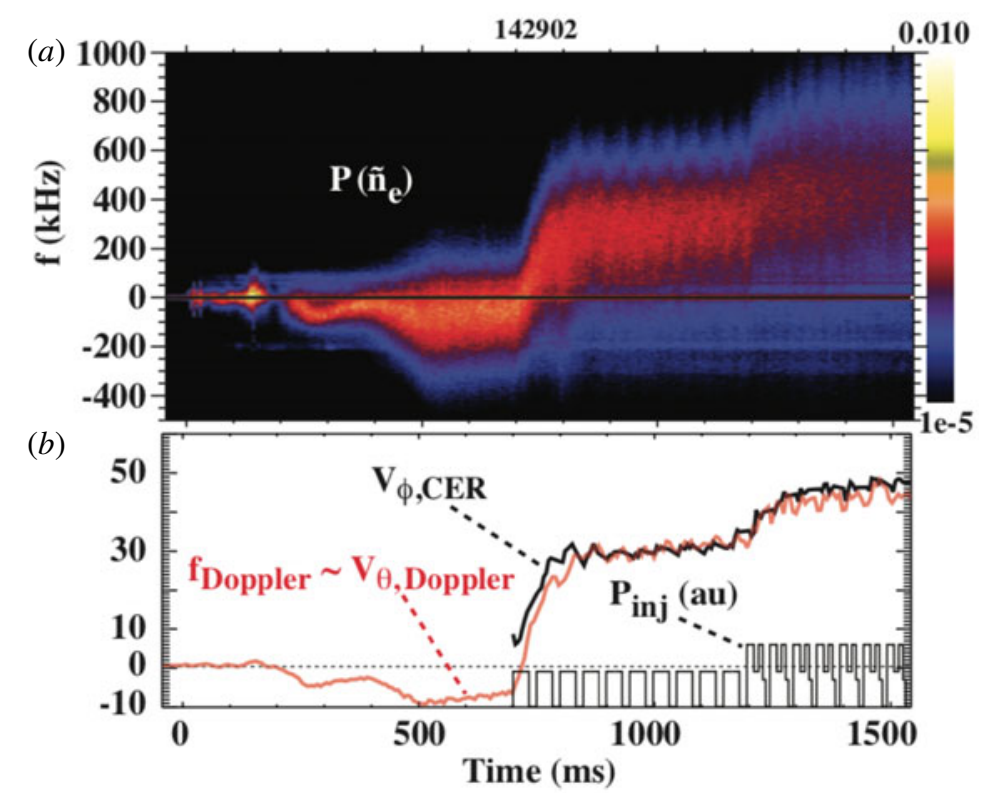

FIgURE 12. (a) The Doppler reflectometer (Doppler backscattering) signal versus time and frequency shows response to changes in rotation associated with neutral beam injection. (b) The mean Doppler shifted frequency (red) is proportional to the phase speed and tracks well the plasma speed measured with charge exchange measurements (CER). Also shown is injected neutral beam power in arbitrary units. From figure 6 of Rhodes et al. (2010).

plasma frame, $\boldsymbol{v}$ is the phase velocity of the plasma and $\boldsymbol{k}_{\boldsymbol{o}}$ is the wavenumber of the measured turbulence. Doppler reflectometers are used to measure the phase velocity of the plasma, as shown in figure 12. The location of the peak of the spectrum changes in response to changes in rotation associated with neutral beam injection in this DIII-D plasma.

The area under the shifted peak of the measured frequency spectrum is integrated to recover the scattered power at the measured turbulent $k_{\mathrm{o}}$. By varying the angle at which the probe beam is launched at the plasma on a shot by shot basis, a Doppler reflectometer can be used to measure a partial wavenumber spectrum of density fluctuations. An example of this is measurement from the TJ-K stellarator is shown in figure 13. The wavenumber spectrum measured at two different radial positions are compared (Fernandez-Marina, Estrada \& Blanco 2014).

Doppler reflectometers maximize the wavenumber resolution by minimizing the spread, $\Delta k$, about the probed wavenumber, $k_{\mathrm{o}}$ (Happel, Blanco \& Estrade 2010; Rhodes et al. 2010). The wavenumber resolution $\Delta k$ can be estimated based on the known width of the probe beam electric field profile, which is selected by the design of the focusing optics, and is typically Gaussian in shape (Hirsch et al. 2001).

One challenge with scattering measurements, including Doppler reflectometry, is the interpretation of the scattered power amplitude as the density fluctuation amplitude. In most cases, full wave simulations are required to extract absolute density fluctuation levels from reflectometry measurements (Nazikian et al. 2001; Conway et al. 2002). Given this additional rather extensive modelling requirement, the Doppler reflectometer spectra and fluctuation levels are typically reported in many experiments with arbitrary 


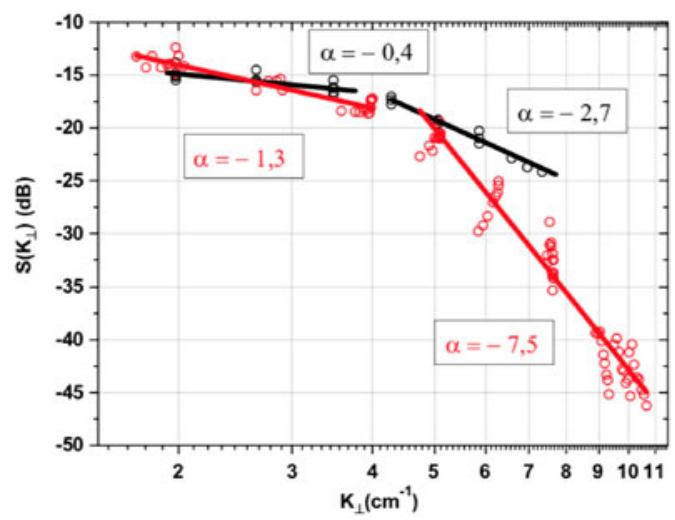

FIGURE 13. Differences in the wavenumber spectrum measured at two different radial positions in a TJ-K plasma with a Doppler reflectometer are shown. From figure 3 of Fernandez-Marina et al. (2014)

units, (a.u.), and comparisons with gyrokinetic simulations have primarily focused on the shape of the frequency spectrum measured with a single Doppler reflectometer channel, or the shape of the wavenumber spectrum, constructed from several channels (or several measurement with the same channel, as the probe angle is varied).

\subsection{Coherent scattering}

Moving to even higher $k$, the electron temperature gradient (ETG) range of wavenumbers, coherent scattering measurements must be used. Diagnostic advances have made it possible to achieve some amount of radial localization diagnostics (Rhodes et al. 2007; Smith et al. 2008; Qing et al. 2010) but unlike CECE, BES and the Doppler reflectometer, which are naturally radially localized based on their diagnostic principles, coherent scattering is limited to measure fluctuations over a three-dimensional scattering volume, and has poorer spatial resolution than the previously discussed measurement techniques. Noting this limitation, measurement of the wavenumber spectrum is possible, using similar data analysis techniques as Doppler reflectometer: the scattered power spectrum from a single channel gives the spectrum of density fluctuations at a particular wavenumber, $k_{\mathrm{o}}$, with resolution $\Delta k$. In the spectrum, a Doppler shift can be seen, centred at the measured $k_{\mathrm{o}}$. By integrating the power spectrum from several channels (several different $k_{\mathrm{o}}$ ) a partial wavenumber spectrum can be reconstructed. An example is shown from NSTX in figure 14. Three channels are used to measure the frequency spectra at three different wavenumbers, and by integrating the power spectra from each channel over the appropriate frequency range, the three-point wavenumber spectrum is obtained.

\section{Synthetic diagnostics}

None of the fluctuation diagnostics used in fusion plasmas can measure the turbulence in plasma frame perfectly. This imperfection manifests in several ways. First, no turbulence diagnostic can cover the full range of turbulent wavenumbers (the low- $k$ ITG/TEM range to the intermediate and high- $k$ range end for TEM/ETG) using a single measurement technique. Each diagnostic samples the turbulence either at the ion scale, the intermediate scale, or the electron scale. Second, each 
(a)
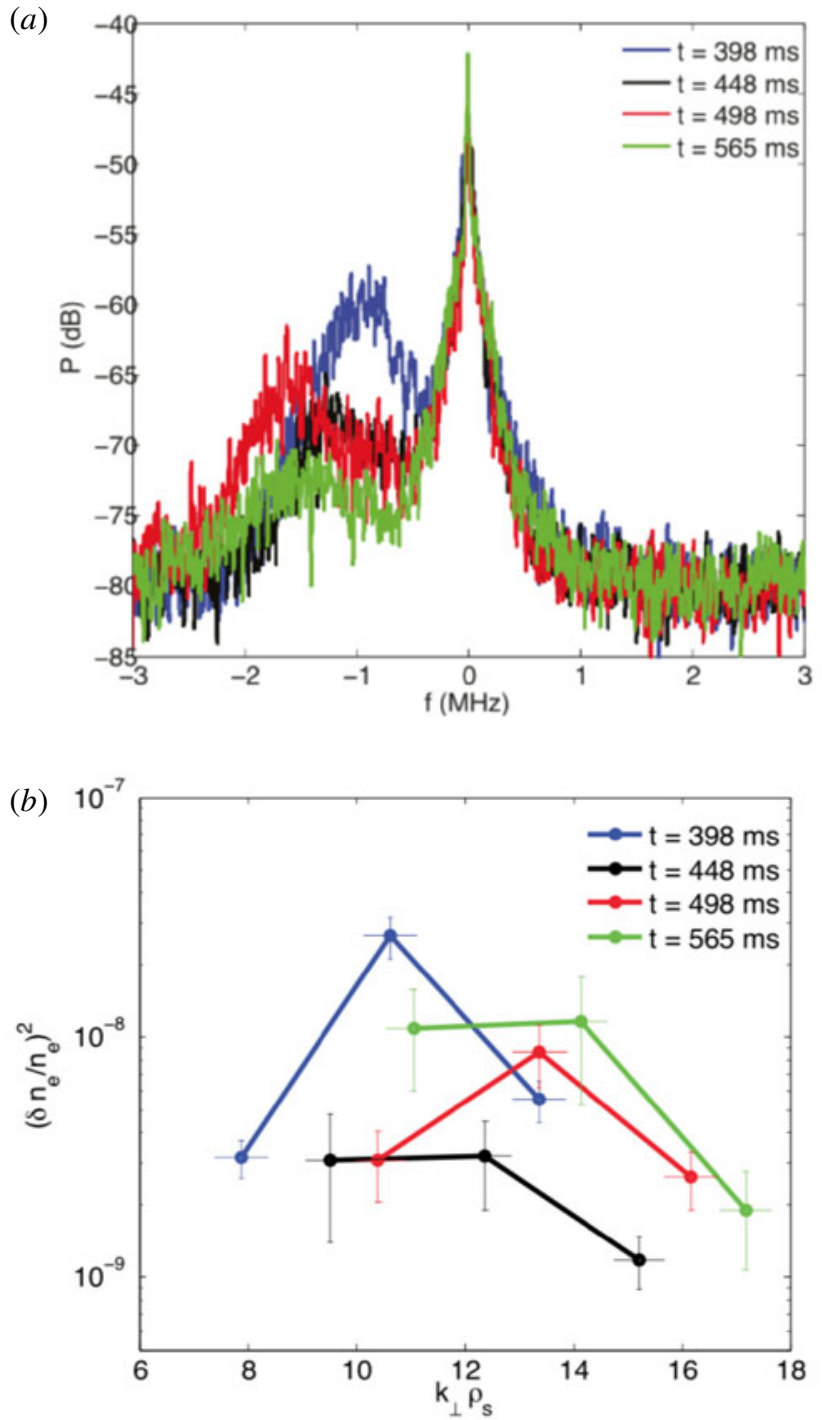

FIgURE 14. From figures 3(b) and 7 of Ruiz Ruiz et al. (2015). (a) Spectra of high- $k$ density fluctuations from channels 1, 2 and 3 of shot 141767 of the high- $k$ scattering system at NSTX. Each channel measures a different turbulent wavenumber. $(b)$ The multichannel data is used to construct the wavenumber spectrum of the measured fluctuations at several different times during the discharge, using the same method as that described for multi-channel Doppler reflectometer data analysis.

diagnostic has limited spatial and temporal resolution, so all reported turbulence measurements represent only an average value. The finite spatial extent of the diagnostic's measurement region is referred to as the 'sample volume' or the 'spot size'. Third, all measurements are made in the laboratory frame - and the plasma is rotating at non-negligible speeds (even without neutral beam injection, intrinsic toroidal rotation can reach substantial Mach numbers many plasmas (Rice 2016)). 


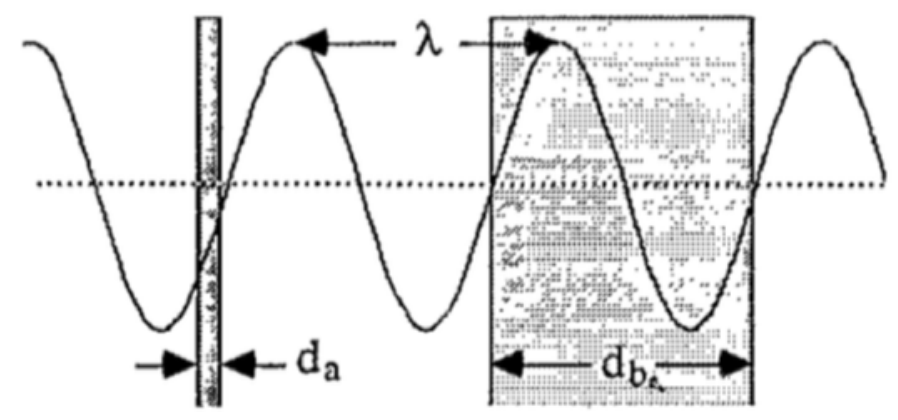

FIGURE 15. From figure 1 of Bravenec \& Wootton (1995). A sketch illustrating sample volume attenuation of a plane wave of wavelength $\mathrm{A}$. The shaded regions of length $d_{a}$ and $d_{b}$ represent 1-D sharp boundaries of the sample volumes or spot sizes.

The measured spectra (even the BES and CECE spectra) will include effects of Doppler shifts that must be quantified and understood. Theory predicts that different types of turbulence will have different frequencies and distinct propagation directions depending on the type of turbulence (Horton 1999). This means it is highly desired, but generally very difficult, to extract the plasma-frame spectrum of the turbulence from measurements. Because a dominant Doppler shift typically determines the shape of the measured spectrum, one must proceed with extreme caution when trying to identify the turbulence based on the laboratory-frame frequency spectrum.

In order to take all these effects into account, one can model the diagnostic response and attempt to unfold how the actual turbulence spectrum would appear in a gyrokinetic simulation (in the plasma frame), given the measurement's limitations. One example of this approach is found in Shafer et al. (2012). It is more common to proceed in the opposite direction: model the diagnostic response and apply the model to the output of a gyrokinetic simulation in order to generate 'synthetic diagnostic data' representative of the laboratory-frame measurements, and then, compare this synthetic data to the actual data from the experiment.

\subsection{Fundamental principles of synthetic diagnostics}

One of the first general descriptions of the effects of the sample volume on turbulence measurements was presented by Bravenec \& Wootton (1995). Because diagnostics such as heavy-ion beam probe (HIBP), beam-emission spectroscopy (BES), correlation electron cyclotron emission (CECE) radiometers and even Langmuir probes, will sample turbulence in a finite volume of space, the measurements will average over shorter-wavelength components of the turbulence. The result is that the measured power spectrum, fluctuation amplitude and correlation lengths will all depend on the size of the sample volume.

Figure 15 shows the effect of spatial averaging on the measurement of a coherent sine wave perturbation and how this is related to wavenumber averaging. If the spot size is less than the wavelength, then the perturbation can be measured, but if the spot size is equal to the wavelength, the peak and trough cancel each other out and the perturbation is not measured. A simple expression, relevant for measurements like $\mathrm{BES}$ and CECE, gives the maximum measureable wavenumber as $k_{\max }=2 \pi / d$, where $d$ is the diameter of the spot size (Bravenec \& Wootton 1995). 

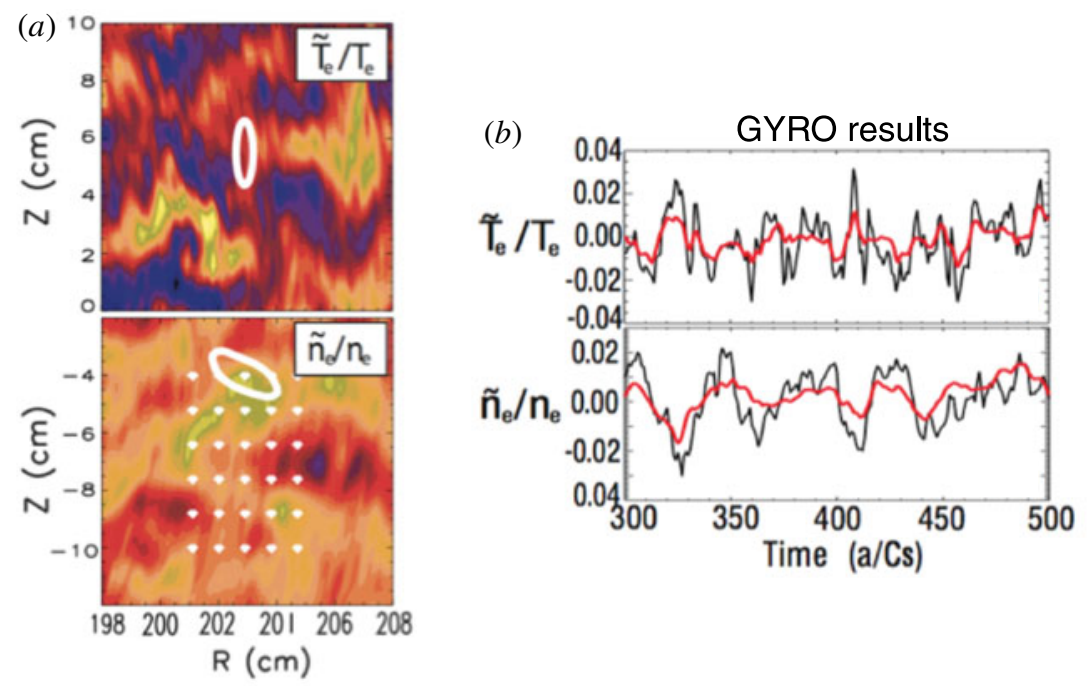

FIGURE 16. (a) Shows the output fluctuations of an ion-scale nonlinear gyrokinetic simulation, mapped from the simulation geometry to real-space geometry in the $R$ and $Z$ plane. The white contour marks the spot size and measurement location a channel from a turbulence diagnostic. (b) The simulated time histories of the fluctuations generated at the centre of the white contours, which mimics the laboratory-frame measurement by including the Doppler shift effects with (red) and without (black) the finite sample volume filter applied.

The numerical implementation steps required to generate synthetic diagnostics for BES and CECE measurements for the gyrokinetic code GYRO are described in Holland et al. (2009), with results illustrated in figure 16. Figure 16(a) shows the output fluctuations of an ion-scale nonlinear gyrokinetic simulation, which have been mapped from the simulation geometry to real-space geometry in the $R$ and $Z$ plane, where $R$ is the major radius of the plasma and $Z$ is the position above the outboard midplane of the tokamak. The white contour marks the spot size and measurement location of one channel of each the CECE and BES diagnostics. A simulated time history of the fluctuations has been generated from the simulation output at the centre of the white contours, without the spatial filtering, is shown in black in figure $16(b)$. The synthetic model includes the Doppler shift effects. After the spatial averaging is applied, the fluctuations within the white contour sample volume are averaged to give the filtered time history (red). The effect of the spatial averaging translates into a low-pass filter on the Doppler-shifted fluctuating synthetic time series, as well as a low-pass filter in $k$-space, as discussed previously (Bravenec \& Wootton 1995).

\subsection{General effects of applying synthetic diagnostics to gyrokinetic simulation outputs}

Ultimately, every synthetic turbulence diagnostic must account for the limited spatial and wavenumber sensitivity of the actual measurements.

Using results from BES synthetic diagnostic, several general examples of how a synthetic diagnostic modifies the raw turbulence predicted by a gyrokinetic code can be described. First, applying a synthetic diagnostic will generally lead to a reduction 

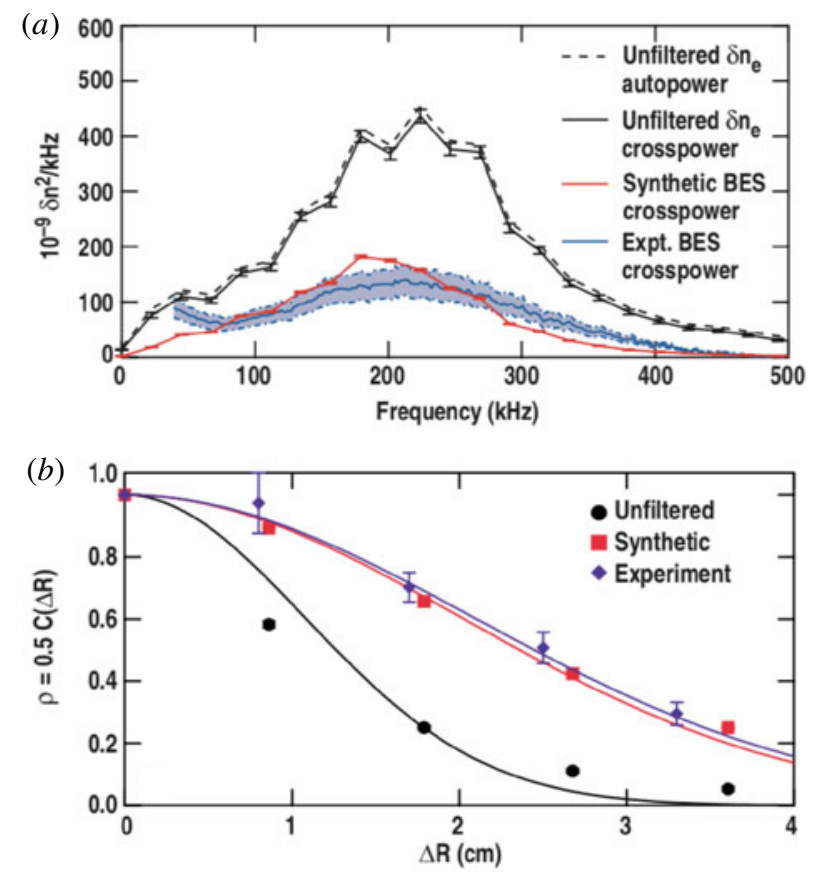

FIGURE 17. Figure shows the reduction in amplitude of the power spectrum $(a)$ and the increase in correlation length $(b)$ due to finite sample volume effects for BES measurements at mid-radius in DIII-D. Panel $(a)$ from figure 7(a) and $(b)$ from figure 12(a) of Holland et al. (2009).

of fluctuation amplitude and an increase in radial correlation length, compared to unfiltered gyrokinetic simulation outputs.

Figure 17(a) demonstrates such effects. The reduction in fluctuation amplitude can be seen in the difference between the unfiltered power spectra and filtered power spectra in figure 17(a), because the r.m.s. fluctuation amplitude is related directly to the area under the curve. The unfiltered power spectrum output by the simulation contains contributions from all simulated wavenumbers and frequencies. The red curve is the power spectrum after application of the synthetic diagnostics. The synthetic diagnostic has filtered the fluctuations, to extract only the measured wavenumber and frequency ranges. This leads to a signal with a reduced r.m.s. fluctuation level, and the reduced spectrum as shown in figure 17(a). The experimental spectrum is shown in blue. The effects of the Doppler shift to set the peak and width of the measured spectrum and the averaging effects of spot size to set the amplitude are needed in the synthetic diagnostic model in order to directly compare the BES power spectrum to a gyrokinetic simulation.

Figure 17(b) shows the effect of the synthetic diagnostic on the correlation length. Because the synthetic diagnostic is acting as a low-pass filter in $k$-space, it attenuates the higher $k$ (longer wavelength) contributions. Also, in this case, it causes the measurement volumes to overlap. The overall effect is an increase in the correlation length after the synthetic diagnostic is applied. This effect must be taken into account to compare the simulation output quantitatively with the measurements.

In addition to general effects, each measurement technique will require a specialized synthetic diagnostic model, which will be dependent on fundamental measurement 


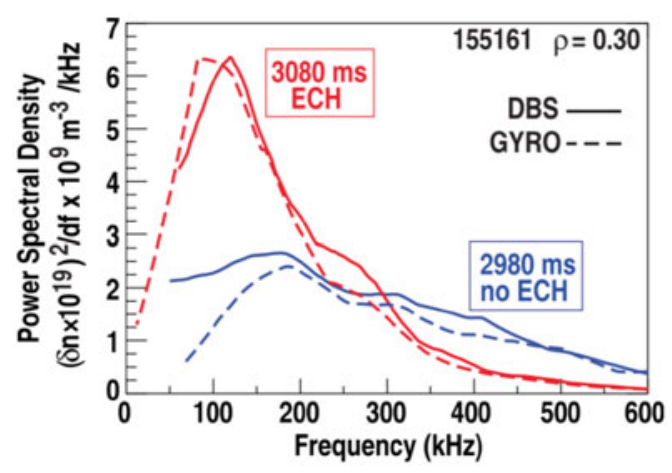

FIGURE 18. From figure 16 of Ernst et al. (2016). The frequency spectra measured at one wavenumber at two different times in a discharge with Doppler backscattering (DBS) at DIII-D are compared to GYRO results by applying a synthetic diagnostic.

principles and practical implementation. For example, with BES and CECE the wavenumber sensitivity is directly linked to the spot size or sample volume (Bravenec \& Wootton 1995; Holland et al. 2009; Shafer et al. 2012).

Synthetic diagnostics for Doppler reflectometers have been implemented for several codes, including the flux-driven code ELMFIRE (Leerink et al. 2010), and the gradient-driven code GYRO (Ernst et al. 2016), with an example comparing a synthetic spectrum from the GYRO code to an experimental spectrum from DIII-D is shown in figure 18 (Ernst et al. 2016). Synthetic diagnostics for high- $k$ coherent scattering measurements have been implemented in GTS (Poli et al. 2010). In the case of Doppler reflectometry measurements, calculation of the wavenumber response and sample volume size is more complicated recent work has focused on advanced synthetic diagnostic modelling that involves full wave simulations of the reflectometer-plasma interactions (Hacquin et al. 2016; Happel et al. 2017).

\subsection{Practical considerations for synthetic diagnostics}

All of the synthetic diagnostics described above are applied during post-processing, i.e. the gyrokinetic code outputs are used to create synthetic time series, and then these time series can be analysed using the exact same analysis techniques as applied to the experimental time series. This has the advantage of allowing a very direct comparison with experiment by using identical analysis routines. However, there are practical challenges to this direct approach. This approach requires fluctuation outputs from the codes at enough spatial and temporal points to reconstruct a time history of the fluctuations in real space $(R, Z)$. This means that the fluctuations from the code must be output at frequent enough time internals to avoid aliasing in frequency space (i.e. the Nyquist frequency of the synthetic time series must be high enough). In addition, the data in real space must be output over a large enough spatial region (simulation box size) and on a fine enough spatial grid to avoid aliasing in $k$-space (Holland et al. 2009; Görler et al. 2014). These requirements result in very large output files from the gyrokinetic simulations, easily exceeding tens of GB, and approaching TBs in some cases. Ideas for in situ synthetic diagnostics are under development, but these are not yet widely available. It is also important to keep in mind that any synthetic diagnostic is fundamentally another model that must be tested, verified and validated independently - ideally before being used with a gyrokinetic code to compare with experiments. 


\section{Frontiers in gyrokinetic transport model validation}

At the frontiers of validation of turbulent transport in fusion plasmas, a growing number of researchers are actively deploying high-performance computing resources for massive simulations of unprecedented physics fidelity. They are also leveraging reduced models in innovative ways using advanced algorithms, artificial intelligence and machine learning; and are pushing the envelope of predictive experimental design initiatives, potentially finding a way to control and optimize turbulent transport. The standard models of drift-wave turbulent transport are being extended to include the interactions between MHD modes and turbulence (McDevitt \& Diamond 2006; Militello et al. 2008; Bañón Navarro et al. 2017) and the interactions between fast ion modes and turbulence (Estrada Mila et al. 2006; Pueschel et al. 2012; Wilkie et al. 2016). Most exciting perhaps, there are experimental observations that can be viewed as grand challenges to the standard turbulent-transport models, including intrinsic rotation and intrinsic rotation reversals (Parra \& Barnes 2015; Rice 2016), momentum transport (Diamond et al. 2013) and 'non-local' perturbative transport phenomena (Ida et al. 2015; Rodriguez-Fernandez et al. 2018a). These areas are described briefly in the next four subsections.

\subsection{Global effects and flux-driven simulations}

The potential impact of 'global effects' on turbulent transport is a frontier area to be explored. Global effects are sometimes also called 'non-local' effects or 'meso-scale' effects. The study of global or non-local effects, such as turbulence spreading or longrange correlations and avalanche type events can be assessed using global, flux-driven simulations (Garbet 2010). It is not possible to provide a full description of this very diverse area of study, even within the limited context of gyrokinetic model validation. Two review articles on 'non-local' phenomena in fusion plasmas are Callen \& Kissick (1997), Ida et al. (2015).

In a flux-driven simulation, where background profiles are evolved along with the turbulence, the validation workflow example described in $\S 3$ is modified. Instead of pressure profiles being used as an input to the code, the heat fluxes are used as an input. The flux-driven simulation then calculates the pressure profiles (due to turbulent transport) and these can be compared with the experiment. Examples of a full- $f$, global gyrokinetic codes run with a flux-driven workflow and compared with turbulence measurements include references (Leerink et al. 2010; Dif-Pradalier et al. 2015; Grandgirard et al. 2016). Flux-driven simulations can be performed with $\partial f$ codes as well. This is done by coupling local gyrokinetic simulations (e.g. flux-tube simulations) to a transport solver. Two examples of this approach are the TRINITY framework (Barnes et al. 2010) and the TGYRO framework (Candy et al. 2009).

It has been suggested that using flux-driven simulations might eliminate some of the complications with extreme sensitivity to inputs used in simulations. A flux-driven simulation uses the experimental fluxes as inputs, so the results will still naturally be sensitive to errors and uncertainties on the inputs (in this case, the input fluxes). The experimentally inferred fluxes depend on the models and measurements used to ascertain the sources and sinks. As described in $\S 3$, the sources and sinks used to calculate the experimental fluxes using power balance code are not without uncertainty. Hence the inputs to the flux-driven simulation will have (sometimes large) error bars and uncertainty, just as inputs to a gradient-driven simulation have (sometimes large) error bars and uncertainties. It would still be necessary to deploy a validation methodology to enable comparison with experiment, which would include 
extensive sensitivity scans of input parameters within experimental error bars. This can become prohibitively expensive when running a global gyrokinetic simulation, where the equilibrium profiles and fluctuations are solved for self-consistently. This is one practical reason why to date very few validation studies have been carried out with global gyrokinetic codes, making this area an exciting frontier.

\subsection{Predictive experimental design: the predict first initiative}

Nonlinear gyrokinetic simulations are usually run after an experiment. In a few cases, they have been run prior to an experiment, in order to design and predict what would be measured. The example discussed in $\S 6.3$ shows how using outputs from a multi-scale simulation to predict changes in turbulence before measuring them in an experiment and is one example of the 'predict first' initiative, or predictive experimental design (Mantica et al. 2017). Documenting code predictions in advance is one additional validation 'best practice' (Greenwald 2010), but can be challenging to do with nonlinear gyrokinetic simulations from a practical perspective. Progress has been made by careful coordination among experiment and theory groups to save and analyse turbulence outputs from simulations that were run for past validation experiments. These outputs can be used before new experiments to select discharge parameters of interest; and the computing resources serve double duty for both interpretation and prediction. This was done in the case of $n-T$-phase angle predictions at DIII-D and AUG (White et al. 2010a; Bañón Navarro et al. 2015; Freethy et al. 2018). An example combining the predict first frontier and simulation of global effects comes from TORESUPRA, where flux-driven, global gyrokinetic simulations have been used to predict the occurrence of so-called 'meso-scale' phenomena prior to experimental observations Dif-Pradalier et al. 2015. Predict first approaches have also been used to provide constraints on hardware designs for new turbulence measurements at C-Mod (White et al. 2011). In addition, nonlinear gyrokinetic simulations have been used to predict turbulence characteristics in the W7-X stellarator years before first plasma operation (Xanthopoulos et al. 2007). Controlling and optimizing turbulent transport in stellarators have even been suggested (Xanthopoulos et al. 2016). It is notable that W7-X has already several turbulence diagnostics (PCI, CECE, etc.), available in the first experimental campaigns.

\subsection{Challenges of validating multi-scale gyrokinetic simulations}

Most gyrokinetic simulations to date exploit the large spatio-temporal scale separation between the ITG and ETG turbulence. This allows one to run single-scale simulations, where either the long wavelength turbulence or the short-wavelength turbulence is simulated. In many cases, high physics fidelity single-scale simulations have been able to reproduce within error bars the measured turbulence characteristics and inferred heat fluxes from power balance calculations in a variety of plasmas across many machines (White et al. 2008; Casati et al. 2009; Holland et al. 2009; White et al. 2010a; Holland et al. 2012 (H-mode); Howard et al. 2013; Told et al. 2013; Field et al. 2014; Görler et al. 2014; White et al. 2015; Sung et al. 2016; Creely et al. 2017, van Wyk et al. 2017; Freethy et al. 2018). In addition, single-scale (ion-scale) simulations can also match measured impurity transport within error bars (Howard et al. 2012). Within the last several years, it was found that certain plasmas could not be well described by single-scale gyrokinetic simulations. This led to the pursuit of realistic mass $\left(m_{\mathrm{i}} / m_{\mathrm{e}}=3600\right)$ multi-scale simulations, which include a range of wavenumbers encompassing ITG/TEM as well as ETG simultaneously 


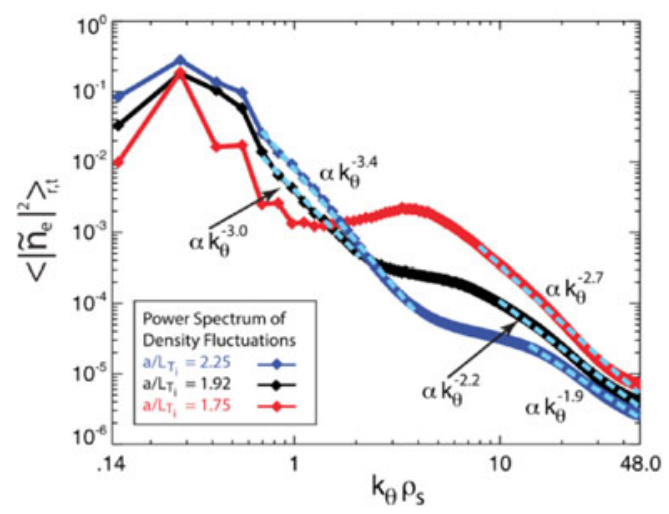

FIgURE 19. From figure 10 Howard et al. (2016). The power spectrum of the density fluctuations is predicted using multi-scale simulations with different values of $a / L T i$. The predicted 'flattening' of the spectrum between $1<k_{\theta} \rho_{s}<5$ (red) compared to the other two cases (black and blue) can be tested experimentally.

(Howard et al. 2014, 2016; Howard 2017; Holland, Howard \& Grierson 2017; Howard et al. 2018). Multi-scale simulations are extremely computationally expensive, and only a handful have been performed to model real experimental discharges. Experimentally realistic multi-scale simulations performed for C-Mod plasmas show expected increases in electron heat flux compared to an ion-scale simulation (contributions from the ETG turbulence). The simulations also found an increase in ion heat flux due to cross-scale interactions, which was not expected (Howard et al. 2014). Cross-scale coupling between ion-scale turbulence and electron-scale turbulence is also seen in Cyclone Base Case simulations (Maeyama et al. 2015); similar enhancements of low- $k$ heat flux were found.

These new physics predictions must be tested by comparing multi-scale simulations with experimental fluctuation measurements. But multi-scale simulations pose several practical challenges for validation. A set of nonlinear ion-scale gyrokinetic simulations used in a typical validation study (including all sensitivity scans) may require up to $5 \mathrm{M}$ CPU hours of computing time. In contrast, a set of nonlinear multi-scale gyrokinetic simulations may require over $100 \mathrm{M} \mathrm{CPU}$ hours for all the scans needed to compare with the experiment. There is a need to leverage fluctuation measurements in new experiments to test existing predictions from multi-scale simulations, because it may be more difficult to run new simulations than to perform new experiments in these cases. In one example of interest, changes in turbulence are predicted in multi-scale simulations that are not seen in ion-scale simulations: the wavenumber spectrum of the intermediate- $k$ turbulence flattens as the $a / L_{\mathrm{Ti}}$ drive is increased, see figure 19 reproduced from Howard et al. (2016). These predictions may be tested using Doppler reflectometer to measure the predicted flattening of the wavenumber spectrum at intermediate wavenumbers in future experiments. This type of approach is part of a 'predict first' initiative in the community, and is discussed more in the next section.

\subsection{Leveraging reduced models and advanced algorithms}

The detailed predictions for turbulence described in the previous section are extremely valuable to probing the underlying physics of turbulent transport. However, many 
predictions of interest for designing new fusion devices do not need high-fidelity gyrokinetic models. Thinking now as engineers - we imagine wanting to be able to run reduced models instead of a full physics nonlinear gyrokinetic model to predict experimental performance. Low-fidelity, approximate models are good enough for engineering design and optimization in many cases. Different goals in the areas of physics and engineering are deeply connected in fusion energy research. The validation effort surrounding nonlinear gyrokinetic codes helps to develop deeper physics understanding, and leads to improved reduced models. In a recent demonstration of this, the physics of cross-scale coupling learned from multi-scale GYRO simulations was adopted and implemented in a new reduced TGLF model (Staebler et al. 2016).

The reduced models are incomplete by design, but are efficient to use. This efficiency has been recently pushed to new extremes. Reduced models can now leverage new developments in big data science, machine learning and artificial intelligence, such as deep learning algorithms. For example, 'trained' transport models based on neural network techniques have been developed using the reduced transport models TGLF (Meneghini et al. 2017) and QuaLiKiz (Citrin et al. 2015), using compiled databases of transport model outputs. Both 'trained' models are faster than TGLF and QuaLiKiz respectively, and could be used to predict experimental transport, potentially in real time. Leveraging advanced algorithms is not limited to big data sets. Applying improved optimization techniques to reduced models is one way to probe which model is most correct, and to determine - possibly before a multi-scale gyrokinetic simulation is run - whether that expensive simulation is needed to explain the experimental observations. A new optimization routine, built around TGLF (Rodriguez-Fernandez et al. 2018b) can also help find which of many drive terms are most important to match the experimental heat fluxes within error bars. Once a set of inputs is identified, one could go back and run nonlinear simulations as needed to verify these cases. Hence this tool can be used as a guide for future, targeted nonlinear gyrokinetic validation studies.

\section{Discussion and outlook}

This article has explored validation of nonlinear gyrokinetic simulations using direct comparisons with measured turbulence in fusion plasmas within a well-established and rigorous methodology. The community-wide effort described here is very much an exercise in physics. Accepting validation of nonlinear turbulence models as a physics research tool is a familiar concept in the domain of fluid mechanics (Moin \& Krishnan 1998). But the concept can seem foreign in the broader fusion community, where a great deal of emphasis is naturally placed on predicting fusion performance. This makes sense, of course, because the ultimate purpose of fusion energy research is to develop a working fusion reactor. When that is the goal, any model that agrees with experiments will do, regardless of the physics contained in the model. For example, the $H_{98 y 2}$ parameter is the thermal energy confinement time normalized to an empirically predicted confinement time; the latter comes from a scaling relation developed from a multi-tokamak database of ELMing H-mode plasmas. The $H_{98 y 2}$ parameter is more widely used than any theory-based model for predicting performance in tokamaks, and it was used to design ITER (ITER 1999).

The very close connection between seeking a deeper understanding of turbulent transport in fusion plasmas and the development of predictive capabilities makes transport model validation a rich area of fusion research. It is very natural and 
correct to view validation of nonlinear gyrokinetic turbulent-transport models as an exercise in the scientific method. Careful comparison between experiment and theory are carried out, ultimately to gain a deeper understanding of the fundamental physical processes of interest. The nonlinear gyrokinetic equations used to describe turbulence in fusion plasmas must be solved numerically, and this creates many challenges, and also many opportunities, to study turbulence. Using validation of gyrokinetic transport models as a research tool creates new opportunities to study plasma turbulence with unprecedented detail. And in fusion research, the connection between validation for physics goals and engineering goals can be quite direct. Guided by advances in understanding the turbulence in fusion plasmas, reduced physics turbulent-transport models are actively being developed with the goal of predicting pressure profiles and fusion performance in ITER (Kinsey et al. 2011). These reduced models will become part of integrated simulation frameworks, which will enable whole device modelling (Bonoli \& McInnes 2015).

As the theoretical framework for understanding transport in fusion plasmas advances, comparing new models, or extensions of the standard models, to experimental results via rigorous validation methodology will become increasingly important. Current fusion devices are quite well diagnosed, and new measurements are coming online in every campaign. Major tokamaks and stellarators around the world, as well as small-to-medium scale fusion and basic plasma experiments, can all become excellent platforms for validation-oriented research with enough investment in diagnostics and modelling efforts. Validation research in the fusion community is vibrant and will continue to expand, with efforts dedicated to advancing predictive capability and to advancing fundamental physics understanding in support of ITER and other future burning plasma experiments.

\section{Acknowledgements}

The author is very grateful to their collaborators T. Goerler, M. Greenwald, W. Guttenfelder and C. Holland, for many years of vigorous discussion about validation of GK codes using turbulence measurements, and for sharing their wisdom on this topic. The author is also indebted to the referees and to A. J. Creely, N. T. Howard, J. Rice, P. Rodriguez Fernandez, F. Sciortino and J. Ruiz Ruiz for many critical comments and insightful suggestions that have greatly improved this article.

\section{REFERENCES}

Anderson, J. K., Forest, C. B., Biewer, J. S., SARfF, J. S. \& Wright, J. C. 2004 Equilibrium reconstruction in the madison symmetric torus reversed field pinch. Nucl. Fusion 44, 162.

Antonsen, T. M. \& LANE, B. 1980 Kinetic equations for low frequency instabilities in inhomogeneous plasmas. Phys. Fluids 23, 1205.

Arshad, S. A., Cordey, J. G., McDonald, D. C., Farthing, J., Joffrin, E., von Hellermann, M., RoACh, C. M. \& Svensson, J. 2008 Chapter 11: data validation, analysis, and applications for fusion plasmas. Fusion Sci. Technol. 53 (2), 667-698.

Bañón Navarro, A., BArdóczi, L., CARTer, T. A., Jenko, F. \& Rhodes, T. L. 2017 Effect of magnetic islands on profiles, flows, turbulence and transport in nonlinear gyrokinetic simulations. Plasma Phys. Control. Fusion 59, 034004.

Bañón Navarro, A., Happel, T., Görler, T, Jenko, F., Abiteboul, J., Bustos, A., Doerk, H., TOLD, D. \& ASDEX UPGRADE TEAM 2015 Gyrokinetic studies of core turbulence features in ASDEX Upgrade H-mode plasmas. Phys. Plasmas 22, 042513. 
Barnes, M., Abel, I. G., Dorland, W., Görler, T., Hammet, G. W. \& Jenko, F. 2010 Direct multiscale coupling of a transport code to gyrokinetic turbulence codes. Phys. Plasmas 17, 056109.

Bergerson, W. F., Xu, P., Irby, J. H., Brower, D. L., Ding, W. \& Marmar, E. S. 2012 Far-infrared polarimetry diagnostic for measurement of internal magnetic field dynamics and fluctuations in the C-MOD tokamak (invited). Rev. Sci. Instrum. 83, $10 \mathrm{E} 316$.

Bonoli, P. \& MCINNES, L. 2015 Report of the workshop on integrated simulations for magnetic fusion energy sciences. US DOE Office of Science. https://science.energy.gov/fes/ community-resources/workshop-reports/.

Bourdelle, C., Citrin, J., Baiocchi, B., Casati, A., Cottier, P., Garbet, X., Imbeaux, F. \& JET CONTRIBUTORS 2016 Core turbulent transport in tokamak plasmas: bridging theory and experiment with QuaLiKiz. Plasma Phys. Control. Fusion 58, 014036.

Brambilla, M. 1999 Numerical simulation of ion cyclotron waves in tokamak plasmas. Plasma Phys. Control. Fusion 41, 1.

Bravenec, R. V., Chen, Y., Candy, J., Wan, W. \& Parker, S. 2013 A verification of the gyrokinetic microstability codes GEM, GYRO, and GS 2. Phys. Plasmas 20, 104506.

Bravenec, R. V. \& Wootton, A. J. 1995 Effects of limited spatial resolution on fluctuation measurements (invited). Rev. Sci. Instrum. 66, 802.

BRIZARD, A. J. \& HAHM, T. S. 2007 Foundations of nonlinear gyrokinetic theory. Rev. Modern Phys. 79, 421.

Budny, R. V., Andre, R., Bateman, G., Halpern, F., Kessel, C. E., Kritz, A. \& McCune, D. 2008 Predictions of H-mode performance in ITER. Nucl. Fusion 48, 075005.

BURRell, K. H. 1998 Effects of $E \times B$ velocity shear and magnetic shear on turbulence and transport in magnetic confinement devices. Phys. Plasmas 4, 1499.

Callen, J. D. \& Kissick, M. W. 1997 Evidence and concepts for non-local transport. Plasma Phys. Control. Fusion 39, B173.

Candy, J., Holland, C., Waltz, R. E., Fahey, M. R. \& Belli, E. 2009 Tokamak profile prediction using direct gyrokinetic and neoclassical simulation. Phys. Plasmas 16, 060704.

Candy, J. \& Waltz, R. E. $2003 a$ An Eulerian gyrokinetic-Maxwell solver. J. Comput. Phys. 186, 545.

CAndy, J. \& Waltz, R. E. $2003 b$ Anomalous transport scaling in the DIII-D tokamak matched by supercomputer simulation. Phys. Rev. Lett. 91, 045001.

CaO, Q., LiU, Y., Zhao, H., Zhou, T., Ti, A. \& HU, L. 2016 Quasi-optical design for systems to diagnose the electron temperature and density fluctuations on EAST. Rev. Sci. Instrum. 87 (11), $11 \mathrm{E} 116$.

Casati, A., Gerbaud, T., Hennequin, P., Bourdelle, C., Candy, J., Clairet, F., Garbet, X., Grandgirard, V., Gurcan, D., Heuraux, S. et al. 2009 Turbulence in the TORE SUPRA tokamak: measurements and validation of nonlinear simulations. Phys. Rev. Lett. 102, 165005.

Chilenski, M. A., FAust, I. C. \& Walk, J. R. 2017 eqtools: Modular, extensible, open-source, cross-machine Python tools for working with magnetic equilibria. Comput. Phys. Commun. 210, 155-162.

Chilenski, M. A., Greenwald, M., Marzouk, Y., Howard, N. T., White, A. E., Rice, J. E. \& WALK, J. R. 2015 Improved profile fitting and quantification of uncertainty in experimental measurements of impurity transport coefficients using Gaussian process regression. Nucl. Fusion 55, 023012.

Cima, G., Bravenec, R. V., Wootton, A. J., Rempel, T. D., Gandy, R. F., Watts, C. \& KwON, M. 1995 Core temperature fluctuations and related heat transport in the Texas Experimental Tokamak-Upgrade. Phys. Plasmas 2, 720.

Citrin, J., Jeko, F., Mantica, P., Told, D., Bourdelle, C., Dumont, R., Garcia, J., Haverkort, J. W., Hogeweij, G. M. D., Johnson, T. et al. \& JET-EFDA CONTRIBUTORS 2014 Ion temperature profile stiffness: non-linear gyrokinetic simulations and comparison with experiment. Nucl. Fusion 54, 023008. 
Citrin, J., Breton, S., Felici, F., Imbeaux, F., Aniel, T., Artaud, J. F., Baiocchi, B., Bourdelle, C., Camenen, Y. \& Garcia, J. 2015 Real-time capable first principle based modelling of tokamak turbulent transport. Nucl. Fusion 55, 092001.

Conway, G. D. 2008 Turbulence measurements in fusion plasmas. Plasma Phys. Control. Fusion 50, 124026.

Conway, G. D., Kurzan, B., Scott, B., Holzhauer, E. \& Kaufmann, M. 2002 Coupling of turbulence and reflectometer simulation codes and comparison with experiment. Plasma Phys. Control. Fusion 44, 451.

Conway, G. D., Schirmer, J., Klenge, S., Suttrop, W., Holzhauer, E. \& The ASdeX UPGRADE TEAM 2004 Plasma rotation profile measurements using Doppler reflectometry. Plasma Phys. Control. Fusion 46, 951.

Creely, A. J., Howard, N. T., Rodriguez-Fernandez, P., CaO, N., Hubbard, A. E., Hughes, J. W., Rice, J. E., White, A. E., Candy, J., Staebler, G. M. et al. 2017 Validation of nonlinear gyrokinetic simulations of L- and I-mode plasmas on Alcator C-Mod. Phys. Plasmas 24, 056104.

DANnERT, T. \& JenKo, F. 2005 Gyrokinetic simulation of collisionless trapped-electron mode turbulence. Phys. Plasmas 12, 072309.

DeBoo, J. C., Petty, C. C., White, A. E., Burrell, K. H., Doyle, E. J., Hillesheim, J. C., Holland, C., McKee, G. R., Rhodes, T. L., Schmitz, L. et al. 2012 Electron profile stiffness and critical gradient studies. Phys. Plasmas 19, 082518.

Deng, B. H., Brower, D. L., Cima, G., Domier, C. W., Luhmann, N. C. Jr \& Watts, C. 1998 Mode structure of turbulent electron temperature fluctuations in the Texas experimental tokamak upgrade. Phys. Plasmas 5, 4117.

Diamond, P. H. 2005 Zonal flows in plasma - a review. Plasma Phys. Control. Fusion 47, R35-R161.

Diamond, P. H., Kosuga, Y., Gürcan, Ö. D., Mcdevitt, C. J., Hahm, T. S., Fedorczak, N., Rice, J. E., WAng, W. X., Ku, S., Kwon, J. M. et al. 2013 An overview of intrinsic torque and momentum transport bifurcations in toroidal plasmas. Nucl. Fusion 53, 104019.

Dif-Pradalier, G., Hornung, G., Ghendrih, P. H., Sarazin, Y., Clairet, F., Vermare, L., Diamond, P. H., Abiteboul, J., Cartier-Michaud, T. et al. 2015 Finding the elusive $E \times B$ staircase in magnetized plasmas. Phys. Rev. Lett. 114, 085004.

Dimits, A. M., Bateman, G., Beer, M. A., Cohen, B. I., Dorland, W., Hammett, G. W., Kim, C., Kinsey, J. E., Kotschenreuther, M., Kritz, A. H. et al. 2000 Comparisons and physics basis of tokamak transport models and turbulence simulations. Phys. Plasmas 7, 969.

Donne, A. J. H., Costley, A. E., Barnsley, R., Bindslev, H., Boivin, R., Conway, G., Fisher, R., Giannella, R., Hartfuss, H., Von Hellermann, M. G. et al. 2007 Chapter 7: Diagnostics. Nucl. Fusion 47, S337-S38.

Doyle, E. J., Houlberg, W. A., Kamada, Y., Mukhovatov, V., Osborne, T. H., Polevoi, A., Bateman, G., Connor, J. W., Cordey, J. G., Tujita, T. et al. 2007 Chapter 2: Plasma confinement and transport. Nucl. Fusion 47, S18-S127.

Ennever, P., Porkolab, M., Candy, J., Staebler, G., Reinke, M. L., Rice, J. E., Rost, J. C., Ernst, D., Hughes, J., Baek, S. G. \& The Alcator C-Mod Team 2016 The effects of main-ion dilution on turbulence in low $q_{95}$ C-Mod ohmic plasmas, and comparisons with nonlinear GYRO. Phys. Plasmas 23, 082509.

Ernst, D. R., Burrell, K. H., Guttenfelder, W., Rhodes, T. L., Dimits, A. M., Bravenec, R., Grierson, B. A., Holland, C., Lohr, J., Marinoni, A. et al. \& The DiII-D team 2016 Role of density gradient driven trapped electron mode turbulence in the H-mode inner core with electron heating. Phys. Plasmas 23, 056112.

Estrada Mila, C., CANDY, J. \& WAltz, R. 2006 Turbulent transport of alpha particles in reactor plasmas. Phys. Plasmas 13, 112303.

Evensen, H. T., Fonck, R. J., Paul, S. F., Rewoldt, G., Scott, S. D., Tang, W. M. \& ZARnStORFF, M. C. 1998 Measurements of ion temperature fluctuations in the tokamak fusion test reactor. Nucl. Fusion 38, 237. 
Fernandez-Marina, F., Estrada, T. \& Blanco, E. 2014 Turbulence radial correlation length measurements using Doppler reflectometry in TJ-II. Nucl. Fusion 54, 072001.

Field, A. R., Dunai, D., Ghim, Y-C., Hill, P., McMillan, B., Roach, C. M., SaArelma, S., Schekochinin, A. A., Zoletnik, S. \& The MAST team 2014 Comparison of BES measurements of ion-scale turbulence with direct gyro-kinetic simulations of MAST L-mode plasmas. Plasma Phys. Control. Fusion 56, 025012.

Fischer, R. \& Dinklage, A. 2007 The concept of integrated data analysis of complementary experiments. AIP Conf. Proc. 954, 195.

Fonck, R. J., DARrow, D. S. \& JAEhnig, K. P. 1984 Determination of plasma-ion velocity distribution via charge-exchange recombination spectroscopy. Phys. Rev. A 29 (6), 3288.

Fontana, M., Porte, L. \& Cabrera, P. M. 2017 Correlation electron cyclotron emission diagnostic in TCV. Rev. Sci. Instrum. 88, 083506.

Forster, J. C., SсHOCh, P. M., НicкоK, R. L. \& Jennings, W. Z. 1994 Study of density and potential fluctuations in the TEXT tokamak with a heavy ion beam probe. IEEE Trans. Plasma Sci. 22 (4), 359.

Freethy, S. J., Conway, G. D., Classen, I., Creely, A. J., Happel, T., Koehn, A., Vanovac, B. \& White, A. E. 2016 Measurement of turbulent electron temperature fluctuations on the ASDEX Upgrade tokamak using correlated electron cyclotron emission. Rev. Sci. Instrum. 87, $11 \mathrm{E} 102$.

Freethy, S. J., Görler, T., Creely, A. J., Conway, G. D., Happel, T., White, A. E. \& THE ASDEX UPGRADE TEAM 2018 Validation of ion-scale gyrokinetic simulations with measurements of electron temperature fluctuations and density-temperature phase angles on ASDEX Upgrade. Phys. Plasmas 25, 055903.

Garbet, X., Mantica, P., Ryter, F., Cordey, G., Imbeaux, F., Sozzi, C., Manini, A., Asp, E., Parail, V., Wolfe, R.\& The JeT EFDA Contributors 2004 Profile stiffness and global confinement. Plasma Phys. Control. Fusion 46, 1351.

GARBET, X. et al. 2010 Gyrokinetic simulations of turbulent transport. Nucl. Fusion 50, 043002.

Garcia, J., Challis, C., Citrin, J., Doerk, H., Giruzzi, G., Görler, T., Jenko, F., Maget, P. \& JET CONTRIButors 2015 Key impact of finite-beta and fast ions in core and edge tokamak regions for the transition to advanced scenarios. Nucl. Fusion 55, 053007.

Görler, T., Lapillone, X., Brunner, S., Dannert, T., Jenko, F., Merz, F. \& Told, E. 2011 The global version of the gyrokinetic turbulence code GENE. J. Comput. Phys. 230, 7053.

Görler, T., White, A. E., Told, D., Jenko, F., Holland, C. \& Rhodes, T. L. 2014 A fluxmatched gyrokinetic analysis of DIII-D L-mode turbulence. Phys. Plasmas 21, 122307.

Grandgirard, V., Abiteboul, J., Bigot, J., Cartier-Michaud, T., Crouseilles, N., DifPradalier, G., Ehrlacher, C. H., Esteve, D., Garbet, X., Ghendrih, P. H. et al. 2016 eqtools: Modular, extensible, open-source, cross-machine Python tools for working with magnetic equilibria. Comput. Phys. Commun. 207, 35-68.

Greenwald, M. 2010 Verification and validation for magnetic fusion. Phys. Plasmas 17, 058101.

Grierson, B. A., Burrell, K. H., Chrystal, C., Groebner, R. J., Kaplan, D. H., Heidbring, W. W., Munoz Burgos, J. M., Pablant, N. A., Solomon, W. M. \& Van ZEELAND, M. A. 2012 Active spectroscopic measurements of the bulk deuterium properties in the DIII-D tokamak (invited). Rev. Sci. Instrum. 83, 10 D529.

Grierson, B. A., Yuan, X., Gorelenkova, M., Kaye, S., Logan, N. C., Meneghini, O., Haskey, S. R., Buchanan, J., Fitzgerald, M., Smith, S. P. et al. 2018 Orchestrating TRANSP simulations for interpretative and predictive tokamak modeling with OMFIT. Fusion Sci. Technol; doi:10.1080/15361055.2017.1398585.

Hacquin, S., Citrin, J., Arnichand, H., Sabot, R., Bourdelle, C., Garbet, X., KramerFleCKen, A. \& THE TORE SUPRA TEAM 2016 Simulation of core turbulence measurement in Tore Supra ohmic regimes. Phys. Plasmas 23, 092303.

Haese, M., Hirsch, M. \& Hartfuss, H. J. 1999 Temperature fluctuations and their correlation with density fluctuations in W7-AS. Rev. Sci. Instrum. 70, 1014.

Happel, T., Blanco, E. \& Estrade, T. 2010 On the role of spectral resolution in velocity shear layer measurements by Doppler reflectometry. Rev. Sci. Instrum. 81, 10 D901. 
Happel, T., Estrade, T., Blanco, E., Tribaldos, V., Cappa, A. \& Bustos, A. 2009 Doppler reflectometer system in the stellarator TJ-II. Rev. Sci. Instrum. 80, 073502.

Happel, T., Görler, T., Hennequin, P., Lechte, C., Bernert, M., Conway, G. D., Freethy, S. J., Honore, C., Pinson, J. R. \& Stroth, U. 2017 Comparison of detailed experimental wavenumber spectra with gyrokinetic simulation aided by two-dimensional full-wave simulations. Plasma Phys. Control. Fusion 59 (5), 054009.

Hawryluk, R. 1979 Close to thermonuclear conditions. In Proceedings of Phys. Plasmas, vol. 1, p. 19. Elsevier.

Hennequin, P., Honore, C., Truc, A., Quemeneur, A. \& Lemoine, N. 2004 Doppler backscattering system for measuring fluctuations and their perpendicular velocity on Tore Supra. Rev. Sci. Instrum. 75, 3881.

Hillesheim, J. C., DeBoo, J. C., Peebles, W. A., Carter, T. A., Wang, G., Rhodes, T. L., Schmitz, L., McKee, G. R., Yan, Z., Staebler, G. M. et al. 2012 2D full wave modeling for a synthetic Doppler backscattering diagnostic. Rev. Sci. Instrum. 83 (10), 10E331.

Hillesheim, J. C., DeBoo, J. C., Peebles, W. A., Carter, T. A., Wang, G., Rhodes, T. L., Schmitz, L., McKee, G. R., Yan, Z., Staebler, G. M. et al. 2013 Observation of a critical gradient threshold for electron temperature fluctuations in the DIII-D tokamak. Phys. Rev. Lett. 110, 045003.

Hillesheim, J. C., Peebles, W. A., Rhodes, T. L., Schmitz, L., Carter, T. A., Gourdain, P.-A. \& WANG, G. 2009 A multichannel, frequency-modulated, tunable Doppler backscattering and reflectometry system. Rev. Sci. Instrum. 80, 083507.

Hillesheim, J. C., Peebles, W. A., Rhodes, T. L., Schmitz, L., White, A. E. \& Carter, T. A. 2010 New plasma measurements with a multichannel millimeter-wave fluctuation diagnostic system in the DIII-D tokamak (invited). Rev. Sci. Instrum. 81, 10 D907.

Hinton, F. L. \& HAzeltine, R. D. 1976 Theory of plasma transport in toroidal confinement systems. Rev. Mod. Phys. 48, 239.

Hirsch, M., Holzhauer, E., Baldzuhn, J., Kurzan, B.\& ScotT, B. 2001 Doppler reflectometry for the investigation of propagating density perturbations. Plasma Phys. Control. Fusion 43, 1641.

Holcomb, C. T., Makowski, M. A., Allen, S. L., Meyer, W. H. \& van Zeeland, M. A. 2008 Overview of equilibrium reconstruction on DIII-D using new measurements from an expanded motional Stark effect diagnostic. Rev. Sci. Instrum. 79, 10F518.

Holland, C. 2016 Validation metrics for turbulent plasma transport. Phys. Plasmas 23, 060901.

Holland, C., Howard, N. T. \& Grierson, B. A. 2017 Gyrokinetic predictions of multiscale transport in a DIII-D ITER baseline discharge. Nucl. Fusion 57, 066043.

Holland, C., Petty, C. C., Schmitz, L., Burrell, K. H., McKee, G. R., Rhodes, T. L. \& CANDY, J. 2012 Progress in GYRO validation studies of DIII-D H-mode plasmas. Nucl. Fusion 52, 114007.

Holland, C., Schmitz, L., Rhodes, T. L., Peebles, W. A., Hillesheim, J. C., Wang, G., Zeng, L., Doyle, E. J., Smith, S. P., Prater, R. et al. 2011 Advances in validating gyrokinetic turbulence models against L- and H-mode plasmas. Phys. Plasmas 18, 056113.

Holland, C., White, A. E., McKee, G. R., Shafer, M. W., Candy, J., Waltz, R. E., SChMitZ, L. \& TYNAN, G. R. 2009 Implementation and application of two synthetic diagnostics for validating simulations of core tokamak turbulence. Phys. Plasmas 16, 052301.

Holzhauer, E., Hirsch, M., Grossman, T., Branaz, B.\& Serra, F. 1998 Theoretical and experimental investigation of the phase-runaway in microwave reflectometry. Plasma Phys. Control. Fusion 40, 1869.

Horton, W. 1999 Drift waves and transport. Rev. Mod. Phys. 71, 735.

Howard, N. T., Greenwald, M., Miknelsen, D. R., Reinke, M. L., White, A. E., Ernst, D., PodPaly, Y. \& CANDY, J. 2012 Quantitative comparison of experimental impurity transport with nonlinear gyrokinetic simulation in an Alcator C-Mod L-mode plasma. Nucl. Fusion 52, 063002 .

Howard, N. T., Holland, C., White, A. E., Greenwald, M. \& Candy, J. 2014 Synergistic cross-scale coupling of turbulence in a tokamak plasma. Phys. Plasmas 21, 112510. 
Howard, N. T., Holland, C., White, A. E., Greenwald, M., Candy, J. \& Creely, A. J. 2016 Multi-scale gyrokinetic simulations: comparison with experiment and implications for predicting turbulence and transport. Phys. Plasmas 23, 056109.

Howard, N. T., Holland, C., White, A. E., Greenwald, M., Rodriguez-Fernandez, P., CANDY, J. \& CREely, A. 2018 Multi-scale gyrokinetic simulations of an Alcator C-Mod, ELM-y H-mode plasma. Plasma Phys. Control. Fusion 60, 014034.

Howard, N. T., White, A. E., Reinke, M. L., Greenwald, M., Holland, C., Candy, J. \& WALK, J. R. 2013 Validation of the gyrokinetic model in ITG and TEM dominated L-mode plasmas. Nucl. Fusion 53 (2013), 123011.

Hutchinson, I. H. 1987 Principles of Plasma Diagnostics. Cambridge University Press.

Hutchinson, I. H. 2002 Principles of plasma diagnostics: second edition. Plasma Phys. Control. Fusion 44, 2603.

Ida, K., Shi, Z., Sun, H. J., Inagaki, S., KamiYa, K., Rice, J. E., Tamura, N., Diamond, P. H., Dif-Pradalier, G., Zou, X. L. et al. 2015 Towards an emerging understanding of non-locality phenomena and non-local transport. Nucl. Fusion 55, 013022.

IshizaWA, A. \& WAELBRoecK, F. L. 2013 Magnetic island evolution in the presence of iontemperature gradient-driven turbulence. Phys. Plasmas 20, 122301.

Ishizawa, A., Maeyama, S., Watanabe, T.-H., Sugama, H. \& Nakajima, N. 2013 Gyrokinetic turbulence simulations of high-beta tokamak and helical plasmas with full-kinetic and hybrid models. Nucl. Fusion 53, 053007.

ITER Physics EXPERT GRoup on CONFInEMEnt And TRAnsport 1999 Chapter 2: Plasma confinement and transport. Nucl. Fusion 39, 2175.

Jenko, F., Dorland, W., Kotschenreuther, M. \& Rogers, B. N. 2000 Electron temperature gradient driven turbulence. Phys. Plasmas 7, 1904.

Jenko, F., Told, D., Görler, T., Citrin, J., Bañón Navarro, A., Bourdelle, C., Brunner, S., Conway, G., Dannert, T., Doerk, H. et al. \& The ASDEX Upgrade Team 2013 Global and local gyrokinetic simulations of high-performance discharges in view of ITER. Nucl. Fusion 53, 073003.

Kasten, C. P., White, A. E. \& Irby, J. 2014 A new fast two-color interferometer at Alcator C-Mod for turbulence measurements and comparison with phase contrast imaging. Phys. Plasmas 21, 042305.

KIM, J., NAM, Y. U., LAMPERT, M. \& GHIM, Y.-C. 2016 Reliability of the two-point measurement of the spatial correlation length from Gaussian-shaped fluctuating signals in fusion-grade plasmas. Nucl. Fusion 56, 106016.

Kinsey, J. E., Staebler, G. M., Candy, J., Waltz, R. E.\& Budny, R. V. 2011 ITER predictions using the GYRO verified and experimentally validated trapped gyro-Landau fluid transport model. Nucl. Fusion 51, 083001.

Kinsey, J. E., WAltZ, R. E. \& CANDY, J. 2006 The effect of safety factor and magnetic shear on turbulent transport in nonlinear gyrokinetic simulations. Phys. Plasmas 13, 022305.

Kinsey, J. E., WAltZ, R. E. \& CANDY, J. 2007 The effect of plasma shaping on turbulent transport and $E \tilde{A} B$ shear quenching in nonlinear gyrokinetic simulations. Phys. Plasmas 14, 102306.

Krommes, J. A. 2010 Nonlinear gyrokinetics: a powerful tool for the description of microturbulence in magnetized plasmas. Phys. Scr. 2010, 014035.

Lao, L. L., Ferron, J. R., Groebner, R. J., Howl, W., St. John, H., Strait, E. J. \& Taylor, T.S. 1990 Equilibrium analysis of current profiles in tokamaks. Nucl. Fusion 30, 1035.

Leerink, S., Bulanin, V. V., Gusakov, E. Z., Heikkinen, J. A., Janhumen, S. J., Kiviniemi, T. P., Korpilo, T., Nora, M. \& OGando, F. 2010 Synthetic doppler reflectometer diagnostic for nonlinear global gyrokinetic simulations. Contrib. Plasma Phys. 50, 242.

Li, G. Q., Ren, Q. L., Qian, J. P., LaO, L. L., Ding, S. Y., Chen, Y. J., Liu, Z. X., Lu, B. \& ZANG, Q. 2013 Kinetic equilibrium reconstruction on EAST tokamak. Plasma Phys. Control. Fusion 55, 125008.

Lin, Z., Hahm, T. S., Lee, W. W., Tang, W. M. \& White, R. B. 1998 Turbulent transport reduction by zonal flows: massively parallel simulations. Science 281, 1835. 
Maeyama, S., Idomura, Y., Wantanabe, T.-H., Nakata, M., Yagi, M., Miyato, N., Ishizawa, A. \& NunAmI, M. 2015 Cross-scale interactions between electron and ion scale turbulence in a tokamak plasma. Phys. Rev. Lett. 114, 255002.

Mantica, P., Bourdelle, C., Camenen, Y., Dejarnac, R., Evans, T., Görler, T., Hillesheim, J., Idomura, Y., Jakubowski, M., Ricci, P. et al. \& The Participants TO THE 21st JOINT EU-US TRANSPORT TASK ForCE WORKShOP 2017 Summary of 21st joint EU-US transport task force workshop (Leysin, September 5-8, 2016). Nucl. Fusion 57, 087001.

Mantica, P., Strintzi, D., Tala, T., Giroud, C., Johnson, T., Leggate, H., Lerche, E., Loarer, T., Peeters, A. G., SAlmi, A. et al. 2009 Experimental study of the ion criticalgradient length and stiffness level and the impact of rotation in the JET tokamak. Phys. Rev. Lett. 102, 175002.

McCarthy, P. J., Martin, P. \& Schneider, W. 1999 The Cliste interpretive equilibrium code. IPP Report 5/85.

McDevitt, C. J. \& Diamond, P. H. 2006 Multiscale interaction of a tearing mode with drift wave turbulence: a minimal self-consistent model. Phys. Plasmas 13, 032302.

McKee, G. R., Burrell, R., Fonck, R., Jackson, G., Murakami, M., Staebler, G., Thomas, D. \& WEST, P. 2000 Impurity-induced suppression of core turbulence and transport in the DIII-D tokamak. Phys. Rev. Lett. 84, 1922.

McKee, G. R., Fonck, R. J., Gupta, D. K., Schlossberg, D. J., Shafer, M. W., Boivin, R. L. \& SOlOMON, W. 2007 Plasma turbulence imaging via beam emission spectroscopy in the core of the DIII-D tokamak. J. Plasma Fusion Res. 2, S1025.

Meneghini, O., Luna, C. J., Smith, S. P. \& LaO, L. L. 2014 Modeling of transport phenomena in tokamak plasmas with neural networks. Phys. Plasmas 21, 060702.

Meneghini, O., Smith, S. P., Snyder, P. B., Staebler, G. M., Candy, J., Belli, E., Lao, L., Kostuk, M., Luce, T., LudA, T. et al. 2017 Self-consistent core-pedestal transport simulations with neural network accelerated models. Nucl. Fusion 57, 086034.

Merlo, G., Dominski, J., Bhattacharjee, A., Chang, C. S., Jenko, F., Ku, S., Lanti, E. \& PARKER, S. 2018 Cross-verification of the global gyrokinetic codes GENE and XGC. Phys. Plasmas 25, 062308.

Mikkelsen, D. R., Howard, N. T., White, A. E. \& Creely, A. J. 2018 Verification of Gene and GYRO with L-mode and I-mode plasmas in Alcator C-Mod. Phys. Plasmas 25, 042505.

Militello, F. L., Waelbroeck, F. L., FitzPatrick, R. \& Horton, W. 2008 Interaction between turbulence and a nonlinear tearing mode in the low beta regime. Phys. Plasmas 15, 050701.

Miller, R. L., Chu, M. S., Greene, J. M., Lin-LiU, Y. R. \& Waltz, R. E. 1998 Noncircular, finite aspect ratio, local equilibrium model. Phys. Plasmas 5, 973.

Moin, P. \& Krishnan, M. 1998 Direct numerical simulation: a tool in turbulence research. Аnnu. Rev. Fluid Mech. 30, 539-578.

Mukhovatov, V. S. \& Shafranov, V. D. 1971 Plasma equilibrium in a tokamak. Nucl. Fusion 11-605.

NaZikian, R., Kramer, G. J. \& VAleo, E. 2001 A tutorial on the basic principles of microwave reflectometry applied to fluctuation measurements in fusion plasmas. Phys. Plasmas 8, 1840.

Nevins, W. M., Candy, J., Cowley, S., Dannert, T., Dimits, A., Dorland, W., EstradaMila, C., Hammett, G. W., Jenko, F., Pueschel, M. J. et al. 2006 Characterizing electron temperature gradient turbulence via numerical simulation. Phys. Plasmas 13, 122306.

Ongena, J. P., Voitsekhovitch, I., Evrard, M. \& McCune, D. 2012 Numerical transport codes. Trans. Fusion Sci. Technol. 61, 180.

Pace, D. C., Austin, M. E., Bass, E. M., Budny, R. V., Heidbrink, W. W., Hillesheim, J. C., Holcomb, C. T., Gorelenkova, M., Grierson, B. A., McCune, D. C. et al. 2013 Energetic ion transport by microturbulence is insignificant in tokamaks. Phys. Plasmas 20, 056108 .

Parra, F. I. \& Barnes, M. 2015 Intrinsic rotation in tokamaks: theory. Plasma Phys. Control. Fusion 57, 045002. 
Pereverzev, G., Yushmanoy, P. N., Dnestrovskit, A. Y., Polevoi, A. R., Tarasian, K. N. \& ZAKHAROV, L. E. 1991 ASTRA automated system of transport analysis in a tokamak. Report of the Mak-Planck-Institut für Plasmaphysik, IPP 5/42.

Petty, C. C., Luce, T. C., DeBoo, J. C., Waltz, R. E., Baker, R. D. \& Wade, M. R. 1998 Scaling of heat transport with beta in the DIII-D tokamak. Nucl. Fusion 38 (8), 1183.

Poli, F. M., Ethier, S., Wang, W., Hahm, T. S., Mazzucato, E. \& Smith, D. R. 2010 A synthetic diagnostic for validation of electron gyroradius scale turbulence simulations against coherent scattering measurements. Phys. Plasmas 17, 112514.

Pueschel, M. J., Jenko, F., Schneller, M., Hauff, T., Gunter, S. \& Tardini, G. 2012 Anomalous diffusion of energetic particles: connecting experiment and simulations. Nucl. Fusion 52, 103018.

Qian, Q., LaO, L. L., Oqilong, R., Baonian, W., Haiqin, L., Long, Z., Zhengping, L., Hanada, K., Dalong, C., Tonghui, S. et al. 2015 Algorithm validation of the current profile reconstruction of EAST based on polarimeter/interferometer. Plasma Sci. Technol. 17 (1), 75.

Qing, Z., Junyu, Z., Li, Y., QIngsheng, H., Yanqing, J., TaO, Z., Xiaoqi, Z., Bhatti, S. H. \& XIANG, G. 2010 Development of a Thomson scattering diagnostic system on EAST. Plasma Sci. Technol. 12, 144.

Rathgeber, S. K., Fischer, R., Fietz, S., Hobirk, J., Kallenbach, A., Meister, H., Putterich, T., Ryter, F., Tardini, G., Wolfrum, E. \& The ASDeX Upgrade Team 2010 Estimation of profiles of the effective ion charge at ASDEX upgrade with integrated data analysis. Plasma Phys. Control. Fusion 52, 095008.

Reinke, M. L., Podpaly, Y. A., Bitter, M., Hutchinson, I. H., Rice, J. E., DelgadoAparicio, L., Gao, C., Greenwald, M., Hill, K., Howard, N. T. et al. 2012 X-ray imaging crystal spectroscopy for use in plasma transport research. Rev. Sci. Instrum. 83 (11), 113504.

Reusch, L. M., Galante, M. E., Franz, P., Johnson, J. R. \& McGarry, M. B. 2014 An integrated data analysis tool for improving measurements on the MST RFP. Rev. Sci. Instrum. 85, $11 \mathrm{D} 844$.

Rhodes, T. L., Holland, C., Smith, S. P., White, A. E., Burrell, K. H., Candy, J., Deboo, J. C., Doyle, E. J., Hillesheim, J. C., Kinsey, J. E. et al. 2011 L-mode validation studies of gyrokinetic turbulence simulations via multiscale and multifield turbulence measurements on the DIII-D tokamak. Nucl. Fusion 51, 063022.

Rhodes, T. L., Leboeuf, J.-N., Sydora, R. D., Groebner, R. J., Doyle, E. J., McKee, G. R., Peebles, W. A., Rittig, C. L., Zeng, L. \& Wang, G. 2002 Comparison of turbulence measurements from DIII-D low-mode and high-performance plasmas to turbulence simulations and models. Phys. Plasmas 9, 2141.

Rhodes, T. L., Peebles, W. A., Crocker, N. A. \& Nguyen, X. 2014 Development of a cross-polarization scattering system for the measurement of internal magnetic fluctuations in the DIII-D tokamak. Rev. Sci. Instrum. 85, $11 \mathrm{D} 838$.

Rhodes, T. L., Peebles, W. A., Nguyen, X., Hillesheim, J. C., Schmitz, L., White, A. E. \& WANG, G. 2010 Quasioptical design of integrated Doppler backscattering and correlation electron cyclotron emission systems on the DIII-D tokamak. Rev. Sci. Instrum. 81 (10), $10 \mathrm{D} 912$.

Rhodes, T. L., Peebles, W. A., Van Zeeland, M. A., Degrassie, J. S., Bravenec, R. V., Burrell, K. H., DeBoo, J. C., Lohr, J., Petty, C. C., Nguyen, X. V. et al. 2007 Response of multiscale turbulence to electron cyclotron heating in the DIII-D tokamak. Phys. Plasmas 14, 056117.

Ricci, P., Theiler, C., Fasoli, A., Furno, I., Gustafson, K., Iraji, D. \& Loizu, J. 2011 Methodology for turbulence code validation: quantification of simulation-experiment agreement and application to the TORPEX experiment. Phys. Plasmas 18, 032109.

RICE, J. E. 2016 Experimental observations of driven and intrinsic rotation in tokamak plasmas. Plasma Phys. Control. Fusion 58, 083001. 
Rodriguez-Fernandez, P., White, A. E., Creely, A. J., Greenwald, M. J., Howard, N. T., Sciortino, F. \& Wright, J. C. $2018 b$ VITALS: a surrogate-based optimization framework for the accelerated validation of plasma transport codes. Fusion Sci. Technol; doi:10.1080/15361055.2017.1396166.

Rodriguez-Fernandez, P., White, A. E., Howard, N. T., Grierson, B. A., Staebler, G. M., Rice, J. E., Yuan, X., CaO, N. M., Greenwald, M. J., Hubbard, A. E. et al. 2018 a Explaining cold-pulse dynamics in tokamak plasmas using local turbulent transport models. Phys. Rev. Lett. 120, 075001.

Rogers, B. N., Drake, J. F. \& ZeIler, A. 1998 Phase space of tokamak edge turbulence, the L-H transition, and the formation of the edge pedestal. Phys. Rev. Lett. 81, 4396.

Ross, D. W. \& Dorland, W. 2002 Comparing simulation of plasma turbulence with experiment. II. Gyrokinetic simulations. Phys. Plasmas 9, 5031.

Ruiz Ruiz, J., Ren, Y., Guttenfelder, W., White, A. E., Kaye, S. M., Leblanc, M. P., Mazzucato, E., Lee, K. C., Domier, C. W., Smith, D. R. et al. 2015 Stabilization of electron-scale turbulence by electron density gradient in national spherical torus experiment. Phys. Plasmas 22, 122501.

Ryter, F., Camenen, Y., DeBoo, J. C., Imbeaux, F., Mantica, P., Regnoli, G., Sozzi, C., Stroth, U. \& ASDEX Upgrade DIII-D, FTU, JET-EFDA Contributors, TCV, TORE SUPRA \& THE W7-AS TEAms 2006 Electron heat transport studies. Plasma Phys. Control. Fusion 48, B453.

SATtLER, S. \& HARTfuss, H. J. 1993 Intensity interferometry for measurement of electron temperature fluctuations in fusion plasmas. Plasma Phys. Control. Fusion 35, 1285-1306.

SATtLeR, S. \& HARTfuss, H. J. 1994 Experimental evidence for electron temperature fluctuations in the core plasma of the W7-AS stellarators. Phys. Rev. Lett. 72, 653.

Shafer, M. W., Fonck, R. J., McKee, G. R., Holland, C., White, A. E. \& Schlossberg, D. J. 2012 2D properties of core turbulence on DIII-D and comparison to gyrokinetic simulations. Phys. Plasmas 19, 032504.

Smith, S. P., Petty, C. C., White, A. E., Holland, C., Bravenec, R., Austin, M. E., Zeng, L. \& Meneghini, O. 2015 Electron temperature critical gradient and transport stiffness in DIII-D. Nucl. Fusion 55, 083011.

Smith, D. R., Mazzucator, E., Lee, W., Park, H. K., Domier, C. W. \& Luhmann, N. C. JR 2008 A collective scattering system for measuring electron gyroscale fluctuations on the national spherical torus experiment. Rev. Sci. Instrum. 79, 123501.

Staebler, G. M., Candy, J., Howard, N. T. \& Holland, C. 2016 The role of zonal flows in the saturation of multi-scale gyrokinetic turbulence. Phys. Plasmas 23 (6), 062518.

Sung, C., White, A. E., Mikkelsen, D. R., Greenwald, M., Holland, C., Howard, N. T., Churchill, R., Theiler, C. \& The Alcator C-Mod Team 2016 Quantitative comparison of electron temperature fluctuations to nonlinear gyrokinetic simulations in C-Mod Ohmic L-mode discharges. Phys. Plasmas 23, 042303.

Terry, P. W., Greenwald, M., Leboeuf, J.-N., McKee, G. R., Mikkelsen, D. R., Nevins, W. M., Newman, D. E. \& Stotler, D. P. 2008 Validation in fusion research: towards guidelines and best practices. Phys. Plasmas 15, 062503.

Tokuszawa, T., EJiri, A., Kawahata, K., Tanaka, K., Yamada, I., Yoshinuma, M., Ida, K. \& SUZUKI, C. 2012 Microwave doppler reflectometer system in LHD. Rev. Sci. Instrum. 83, $10 \mathrm{E} 322$.

Told, D., Jenko, F., Görler, T., Casson, F. J., Fable, E. \& The ASDEX Upgrade Team 2013 Characterizing turbulent transport in ASDEX Upgrade L-mode plasmas via nonlinear gyrokinetic simulations. Phys. Plasmas 20, 122312.

Tynan, G. R., Fujisawa, A. \& MCKee, G. 2009 A review of experimental drift turbulence studies. Plasma Phys. Control. Fusion 51, 113001.

Uzun-Kaymak, I. U., Fonck, R. J. \& MCKeE, G. R. 2012 Ultra-fast charge exchange spectroscopy for turbulent ion temperature fluctuation measurements on the DIII-D tokamak (invited). Rev. Sci. Instrum. 83, 10D526. 
VAezi, P. \& Holland, C. 2018 An improved approach to uncertainty quantification for plasma turbulence validation studies. Fusion Sci. Technol. 74, 77-88.

Viezzer, E., Putterich, T., Dux, R., McDermott, R. M. \& ASDEX Upgrade Team 2012 High-resolution charge exchange measurements at ASDEX Upgrade. Rev. Sci. Instrum. 83, 103501.

Waltz, R., Casati, A. \& Staebler, G. 2009 Gyrokinetic simulation tests of quasilinear and tracer transport. Phys. Plasmas 16, 072303.

Weiland, J. 1999 Collective Modes in Inhomogeneous Plasmas. Institute of Physics Publishing.

White, A. E., Howard, N. T., Creely, A. J., Chilenski, M. A., Greenwald, M., Hubbard, A. E., Hughes, J. W., Marmar, E., Rice, J. E., Sierchio, J. M. et al. 2015 Nonlinear gyrokinetic simulations of the I-mode high confinement regime and comparisons with experiment. Phys. Plasmas 22, 056109.

White, A. E., Howard, N. T., Greenwald, M., Reinke, M. L., Sung, C., Baek, S., Barnes, M., Candy, J., Dominguez, A., Ernst, D. et al. \& Alcator C-Mod Team 2013 Multichannel transport experiments at Alcator C-Mod and comparison with gyrokinetic simulations. Phys. Plasmas 20, 056106.

White, A. E., Howard, N. T., Mikkelsen, D. R., Greenwald, M., Candy, J. \& Waltz, R. E. 2011 Feasibility study for a correlation electron cyclotron emission turbulence diagnostic based on nonlinear gyrokinetic simulations. Plasma Phys. Control. Fusion 53, 115003.

White, A. E., Peebles, W. A., Rhodes, T. L., Holland, C. H., Wang, G., Schmitz, L., Carter, T. A., Hillesheim, J. C., Doyle, E. J., Zeng, L. et al. $2010 a$ Measurements of the cross-phase angle between density and electron temperature fluctuations and comparison with gyrokinetic simulations. Phys. Plasmas 17, 056103.

White, A. E., Schmitz, A. E., McKee, G. R., Holland, C., Peebles, W. A., Carter, T. A., Shafer, M. W., Austin, M. E., Burrell, K. H., CAndy, J. et al. 2008 Measurements of core electron temperature and density fluctuations in DIII-D and comparison to nonlinear gyrokinetic simulations. Phys. Plasmas 15, 056116.

White, A. E., Schmitz, L., Peebles, W. A., Rhodes, T. L., Carter, T. A., McKee, G. R., Shafer, M. W., Staebler, G. M., Burrell, G. M., DeBoo, J. C. et al. $2010 b$ Simultaneous measurement of core electron temperature and density, fluctuations during electron cyclotron heating on DIII-D. Phys. Plasmas 17, 020701.

Whyte, D. G., Hubbard, A. E., Hughes, J. W., Lipschultz, B., Rice, J. E., Marmar, E. S., Greenwald, M., Cziegler, I., Dominguez, A., Golfinopoulos, T. et al. 2010 I-mode: an H-mode energy confinement regime with L-mode particle transport in Alcator C-Mod. Nucl. Fusion 50, 105005.

Wilkie, G. J., Abel, I. G., Landreman, M. \& Dorland, W. 2016 Transport and deceleration of fusion products in microturbulence. Phys. Plasmas 23 (6), 060703.

VAn Wyk, F., Highcock, E. G., Field, A. R., Roach, C. M., Schekochinin, A. A., Parra, F. I. \& DORLAND, W. 2017 Ion-scale turbulence in MAST: anomalous transport, subcritical transitions, and comparison to BES measurements. Plasma Phys. Control. Fusion 59, 114003.

Xanthopoulos, F., Merz, F., Görler, T. \& Jenko, F. 2007 Nonlinear gyrokinetic simulations of ion-temperature-gradient turbulence for the optimized Wendelstein 7-X stellarator. Phys. Rev. Lett. 99, 035002.

Xanthopoulos, P., Mynick, H. E., Helander, P., Turkin, Y., Plunk, G. G., Jenko, F., Görler, T., TOLD, D., Bird, T. \& Proll, J. H. E. 2016 Controlling turbulence in present and future stellarators. Phys. Rev. Lett. 113, 155001.

Zemedkun, S. E., Che, S., Chen, Y., Domier, C. W., Luhmann, N. C., Munsat, T., PARKer, S. E., Tobias, B., WAN, W. \& YU, L. 2015 Spatially resolved measurements of two-dimensional turbulent structures in DIII-D plasmas. Phys. Plasmas 22, 122508.

Zhou, C., Liu, A. D., Zhang, X. H., Hu, J. Q., Wang, M. Y., Li, H., LAN, T., Xie, J. L., Sun, X., Ding, W. X. et al. 2013 Microwave doppler reflectometer system in the experimental advanced superconducting tokamak. Rev. Sci. Instrum. 84, 103511. 